\title{
A Framework for the Computational Linguistic Analysis of Dehumanization
}

\author{
Julia Mendelsohn ${ }^{1 *}$, Yulia Tsvetkov ${ }^{2}$ and Dan Jurafsky ${ }^{3}$ \\ ${ }^{1}$ School of Information, University of Michigan, Ann Arbor, MI, United States, ${ }^{2}$ Language Technologies Institute, Carnegie \\ Mellon University, Pittsburgh, PA, United States, ${ }^{3}$ Department of Linguistics, Stanford University, Stanford, CA, United States
}

\section{OPEN ACCESS}

Edited by:

Jack Grieve,

University of Birmingham,

United Kingdom

Reviewed by:

Wei Lu,

Singapore University of Technology and Design, Singapore Jonathan Schler,

Holon Institute of Technology, Israel

*Correspondence: Julia Mendelsohn juliame@umich.edu

Specialty section: This article was submitted to Language and Computation,

a section of the journal

Frontiers in Artificial Intelligence

Received: 03 March 2020

Accepted: 29 June 2020

Published: 07 August 2020

Citation:

Mendelsohn J, Tsvetkov Y and Jurafsky D (2020) A Framework for the Computational Linguistic Analysis of

Dehumanization.

Front. Artif. Intell. 3:55. doi: 10.3389/frai.2020.00055
Dehumanization is a pernicious psychological process that often leads to extreme intergroup bias, hate speech, and violence aimed at targeted social groups. Despite these serious consequences and the wealth of available data, dehumanization has not yet been computationally studied on a large scale. Drawing upon social psychology research, we create a computational linguistic framework for analyzing dehumanizing language by identifying linguistic correlates of salient components of dehumanization. We then apply this framework to analyze discussions of LGBTQ people in the New York Times from 1986 to 2015. Overall, we find increasingly humanizing descriptions of LGBTQ people over time. However, we find that the label homosexual has emerged to be much more strongly associated with dehumanizing attitudes than other labels, such as gay. Our proposed techniques highlight processes of linguistic variation and change in discourses surrounding marginalized groups. Furthermore, the ability to analyze dehumanizing language at a large scale has implications for automatically detecting and understanding media bias as well as abusive language online.

Keywords: computational sociolinguistics, dehumanization, lexical variation, language change, media, New York Times, LGBTQ

\section{INTRODUCTION}

Despite the American public's increasing acceptance of LGBTQ people and recent legal successes, LGBTQ individuals remain targets of hate and violence (Dinakar et al., 2012; Silva et al., 2016; Gallup, 2019). At the core of this issue is dehumanization, "the act of perceiving or treating people as less than human" (Haslam and Stratemeyer, 2016), a process that heavily contributes to extreme intergroup bias (Haslam, 2006). Language is central to studying this phenomenon; like other forms of bias (Wiebe et al., 2004; Greene and Resnik, 2009; Recasens et al., 2013; Voigt et al., 2017; Breitfeller et al., 2019), dehumanizing attitudes are expressed through subtle linguistic manipulations, even in carefully-edited texts. It is crucial to understand the use of such linguistic signals in mainstream media, as the media's representation of marginalized social groups has far-reaching implications for social acceptance, policy, and safety.

While small-scale studies of dehumanization and media representation of marginalized communities provide valuable insights (e.g., Esses et al., 2013), there exist no known largescale analyses, likely due to difficulties in quantifying such a subjective and multidimensional psychological process. However, the ability to do large-scale analysis is crucial for understanding how dehumanizing attitudes have evolved over long periods of time. Furthermore, by being able to account for a greater amount of media discourse, large-scale techniques can provide a more complete view of the media environment to which the public is exposed. 
Linguistics and computer science offer valuable methods and insights on which large-scale techniques might be developed for the study of dehumanization. By leveraging more information about the contexts in which marginalized groups are discussed, computational linguistic methods enable large-scale study of a complex psychological phenomenon and can even reveal linguistic variations and changes not easily identifiable through qualitative analysis alone.

We develop a computational linguistic framework for analyzing dehumanizing language, with a focus on lexical signals of dehumanization. Social psychologists have identified numerous components of dehumanization, such as negative evaluations of a target group, denial of agency, moral disgust, and likening members of a target group to non-human entities, such as vermin. Drawing upon this rich body of literature, we first identify linguistic analogs for these components and propose several computational techniques to measure these linguistic correlates. We then apply this general framework to explore changing representations of LGBTQ groups in the New York Times over 30 years and both qualitatively and quantitatively evaluate our techniques within this case study. We additionally use this lens of dehumanization to investigate differences in social meaning between the denotationally-similar labels gay and homosexual. We focus on a single case study in order to conduct an in-depth analysis of our methodology, but our framework can generalize to study representations of other social groups, which we briefly explore in our discussion.

This paper aims to bridge the gaps between computational modeling, sociolinguistics, and dehumanization research with implications for several disciplines. In addition to enabling largescale studies of dehumanizing language and media representation of marginalized social groups, these techniques can be built into systems that seek to capture both conscious and unconscious biases in text. Furthermore, this work has implications for improving machines' abilities to automatically detect hate speech and abusive language online, which are typically underpinned by dehumanizing language. Finally, our case study demonstrates that such computational analyses of discussions about marginalized groups can provide unique insights into language variation and change within sensitive sociopolitical contexts, and help us understand how people (and institutions) use language to express their ideologies and attitudes toward certain social groups.

Trigger Warning: this paper contains offensive material that some may find upsetting, especially in Table 4 and Table 7.

\section{BACKGROUND}

\subsection{Dehumanization}

Our lexical semantic analysis involves quantifying linguistic correlates of component psychological processes that contribute to dehumanization. Our approaches are informed by social psychology research on dehumanization, which is briefly summarized here. Prior work has identified numerous related processes that comprise dehumanization (Haslam, 2006). One such component is likening members of the target group to nonhuman entities, such as machines or animals (Haslam, 2006; Goff et al., 2008; Kteily et al., 2015). By perceiving members of a target group to be non-human, they are "outside the boundary in which moral values, rules, and considerations of fairness apply" (Opotow, 1990), which thus leads to violence and other forms of abuse. Metaphors and imagery relating target groups to vermin are particularly insidious and played a prominent role in the genocide of Jews in Nazi Germany and Tutsis in Rwanda (Harris and Fiske, 2015). More recently, the vermin metaphor has been invoked by the media to discuss terrorists and political leaders of majority-Muslim countries after September 11 (Steuter and Wills, 2010). According to Tipler and Ruscher (2014), the vermin metaphor is particularly powerful because it conceptualizes the target group as "engaged in threatening behavior, but devoid of thought or emotional desire."

Disgust underlies the dehumanizing nature of these metaphors and is itself another important element of dehumanization. Disgust contributes to members of target groups being perceived as less-than-human and of negative social value (Sherman and Haidt, 2011). It is often evoked (both in real life and experimental settings) through likening a target group to animals. Buckels and Trapnell (2013) find that priming participants to feel disgust facilitates "moral exclusion of out-groups." Experiments by Sherman and Haidt (2011) and Hodson and Costello (2007) similarly find that disgust is a predictor of dehumanizing perceptions of a target group. Both moral disgust toward a particular social group and the invocation of non-human metaphors are facilitated by essentialist beliefs about groups, which Haslam (2006) presents as a necessary component of dehumanization. In order to distinguish between human and non-human, dehumanization requires an exaggerated perception of intergroup differences. Essentialist thinking thus contributes to dehumanization by leading to the perception of social groups as categorically distinct, which in turn emphasizes intergroup differences (Haslam, 2006).

According to Haslam (2006), prior work describes "extremely negative evaluations of others" as a major component of dehumanization. This is especially pronounced in Bar-Tal's account of delegitimization, which involves using negative characteristics to categorize groups that are "excluded from the realm of acceptable norms and values" (Bar-Tal, 1990). While Bar-Tal's defines delegitimization as a distinct process, he considers dehumanization to be one means of delegitimization. Opotow (1990) also discusses broader processes of moral exclusion, one of which is dehumanization. A closely related process is psychological distancing, in which one perceives others to be objects or non-existent (Opotow, 1990). Nussbaum (1999) identifies elements that contribute to the objectification (and thus dehumanization) of women, one of which is denial of subjectivity, or the habitual neglect of one's experiences, emotions, and feelings.

Another component of dehumanization is the denial of agency to members of the target group (Haslam, 2006). According to Tipler and Ruscher, there are three types of agency: the ability to (1) experience emotion and feel pain (affective mental states), (2) act and produce an effect on their environment (behavioral potential), and (3) think and hold beliefs (cognitive mental states) (Tipler and Ruscher, 2014). Dehumanization typically involves the denial of one or more of these types of agency (Tipler and Ruscher, 2014). 
In section 3, we introduce computational linguistic methods to quantify several of these components.

\subsection{Related Computational Work}

While this is the first known computational work that focuses on dehumanization, we draw upon a growing body of literature at the intersection of natural language processing and social science. We are particularly inspired by the area of automatically detecting subjective language, largely pioneered by Wiebe et al. who developed novel lexical resources and algorithms for this task (Wiebe et al., 2004). These resources have been used as linguistically-informed features in machine learning classification of biased language (Recasens et al., 2013). Other work has expanded this lexicon-based approach to account for the role of syntactic form in identifying the writer's perspective toward different entities (Greene and Resnik, 2009; Rashkin et al., 2016).

These methods have been used and expanded to analyze pernicious, but often implicit social biases (Caliskan et al., 2017). For example, Voigt et al. analyze racial bias in police transcripts by training classifiers with linguistic features informed by politeness theory (Voigt et al., 2017), and Garg et al. investigate historical racial biases through changing word embeddings (Garg et al., 2018). Other studies focus on how people's positions in different syntactic contexts affect power and agency, and relate these concepts to gender bias in movies (Sap et al., 2017) and news articles about the \#MeToo movement (Field et al., 2019). There is also a growing focus on identifying subtle manifestations of social biases, such as condescension (Wang and Potts, 2019), microagressions (Breitfeller et al., 2019), and “othering” language (Burnap and Williams, 2016; Alorainy et al., 2019). In addition, our focus on dehumanization is closely related to the detection and analysis of hate speech and abusive language (Schmidt and Wiegand, 2017; ElSherief et al., 2018).

Gender and racial bias have also been identified within widelydeployed NLP systems, for tasks including toxicity detection (Sap et al., 2019), sentiment analysis (Kiritchenko and Mohammad, 2018), coreference resolution (Rudinger et al., 2018), language identification (Blodgett and O'Connor, 2017), and in many other areas (Sun et al., 2019). Given the biases captured, reproduced, and perpetuated in NLP systems, there is a growing interest in mitigating subjective biases (Sun et al., 2019), with approaches including modifying embedding spaces (Bolukbasi et al., 2016; Manzini et al., 2019), augmenting datasets (Zhao et al., 2018), and adapting natural language generation methods to "neutralize" text (Pryzant et al., 2019).

A related line of research has developed computational approaches to investigate language use and variation in media discourse about sociopolitical issues. For example, some work has drawn upon political communication theory to automatically detect an issue's framing (Entman, 1993; Boydstun et al., 2013; Card et al., 2015) through both supervised classification (Boydstun et al., 2014; Baumer et al., 2015) and unsupervised methods, such as topic modeling and lexicon induction (Tsur et al., 2015; Field et al., 2018; Demszky et al., 2019). Scholars have also developed computational methods to identify lexical cues of partisan political speech, political slant in mass media, and polarization in social media (Monroe et al., 2008; Gentzkow and Shapiro, 2010; Demszky et al., 2019).

\subsection{Attitudes Toward LGBTQ Communities in the United States}

Some background about LGBTQ communities is necessary for our case study of LGBTQ dehumanization in the New York Times. Bias against LGBTQ people is longstanding in the United States. Overall, however, the American public has become more accepting of LGBTQ people and supportive of their rights. In 1977, equal percentages of respondents (43\%) agreed and disagreed with the statement that gay or lesbian relations between consenting adults should be legal (Gallup, 2019). Approval of gay and lesbian relations then decreased in the 1980s; in 1986, only $32 \%$ of respondents believed they should be legal. According to Gallup, attitudes have become increasingly positive since the 1990 s, and in $2019,73 \%$ responded that gay or lesbian relations should be legal. The Pew Research center began surveying Americans about their beliefs about same-sex marriage in 2001 and found similar trends (Pew Research Center, 2017). Between 2001 and 2019, support for same-sex marriage jumped from 35 to $61 \%$.

In addition to the public's overall attitudes, it is important to consider the specific words used to refer to LGBTQ people. Because different group labels potentially convey different social meanings, and thus have different relationships with dehumanization, our case study compares two LGBTQ labels: gay and homosexual. The Gallup survey asked for opinions on legality of "homosexual relations" until 2008, but then changed the wording to "gay and lesbian relations." This was likely because many gay and lesbian people find the word homosexual to be outdated and derogatory. According to the LGBTQ media monitoring organization GLAAD, homosexual's offensiveness originates in the word's dehumanizing clinical history, which had falsely suggested that "people attracted to the same sex are somehow diseased or psychologically/emotionally disordered"1. Beyond its outdated clinical associations, some argue that the word homosexual is more closely associated with sex and all of its negative connotations simply by virtue of containing the word sex, while terms, such as gay and lesbian avoid such connotations (Peters, 2014). Most newspapers, including the New York Times, almost exclusively used the word homosexual in articles about gay and lesbian people until the late 1980s (Soller, 2018). The New York Times began using the word gay in non-quoted text in 1987. Many major newspapers began restricting the use of the word homosexual in 2006 (Peters, 2014). As of 2013, the New York Times has confined the use of homosexual to specific references to sexual activity or clinical orientation, in addition to direct quotes and paraphrases ${ }^{2}$.

Beyond differences in how LGBTQ people perceive the terms gay or lesbian relative to homosexual, the specific choice of label can affect attitudes toward LGBTQ people. In 2012, Smith et al. (2017) asked survey respondents about either "gay and lesbian rights" or "homosexual rights." Respondents who read the word

\footnotetext{
${ }^{1}$ https://www.glaad.org/reference/lgbtq

${ }^{2}$ https://www.glaad.org/reference/style
} 
"homosexual" showed less support for LGBTQ rights. This effect was primarily driven by high authoritarians, people who show high sensitivity to intergroup distinctions. The authors posit that homosexual makes social group distinctions more blatant than gay or lesbian. This leads to greater psychological distancing, thus enabling participants to remove LGBTQ people from their realm of moral consideration (Smith et al., 2017). Based on prior research and evolving media guidelines, we expect our computational analysis to show that homosexual occurs in more dehumanizing contexts than the label gay.

\section{OPERATIONALIZING DEHUMANIZATION}

In section 2.1, we discussed multiple elements of dehumanization that have been identified in social psychology literature. Here we introduce and quantify lexical correlates to operationalize four of these components: negative evaluations of a target group, denial of agency, moral disgust, and use of vermin metaphors.

\subsection{Negative Evaluation of a Target Group}

One prominent aspect of dehumanization is extremely negative evaluations of members of a target group (Haslam, 2006). Attribution of negative characteristics to members of a target group in order to exclude that group from "the realm of acceptable norms and values" is specifically the key component of delegitimization, a process of moral exclusion closely related to dehumanization. We hypothesize that this negative evaluation of a target group can be realized by words and phrases whose connotations have extremely low valence, where valence refers to the dimension of meaning corresponding to positive/negative (or pleasure/displeasure) (Osgood et al., 1957; Mohammad, 2018). Thus, we propose several valence lexicon-based approaches to measure this component: paragraph-level valence analysis, Connotation Frames of perspective, and word embedding neighbor valence. Each technique has different advantages and drawbacks regarding precision and interpretability.

\subsubsection{Paragraph-Level Valence Analysis}

One dimension of affective meaning is valence, which corresponds to an individual's evaluation of an event or concept, ranging from negative/unpleasant to positive/pleasant (Osgood et al., 1957; Russell, 1980). A straightforward lexical approach to measure negative evaluations of a target group involves calculating the average valence of words occurring in discussions of the target group. We obtain valence scores for 20,000 words from the NRC VAD lexicon, which contains real-valued scores ranging from zero to one for valence, arousal and dominance. A score of zero represents the lowest valence (most negative emotion) and a score of one is the highest possible valence (most positive emotion) (Mohammad, 2018). Words with the highest valence include love and happy, while words with the lowest valence include nightmare and shit.

We use paragraphs as the unit of analysis because a paragraph represents a single coherent idea or theme (Hinds, 1977). This is particularly true for journalistic writing (Shuman, 1894), and studies on rhetoric in journalism often treat paragraphs as the unit of analysis (e.g., Barnhurst and Mutz, 1997; Katajamaki and Koskela, 2006). Furthermore, by looking at a small sample of our data, we found that paragraphs were optimal because full articles often discuss unrelated topics while single sentences do not provide enough context to understand how the newspaper represents the target group. We calculate paragraph-level scores by taking the average valence score over all words in the paragraph that appear (or whose lemmas appear) in the NRC VAD lexicon.

\subsubsection{Connotation Frames of Perspective}

While paragraph-level valence analysis is straightforward, it is sometimes too coarse because we aim to understand the sentiment directed toward the target group, not just nearby in the text. For example, suppose the target group is named "B." A sentence, such as "A violently attacked B" would likely have extremely negative valence, but the writer may not feel negatively toward the victim, "B."

We address this by using Rashkin et al.'s Connotation Frames Lexicon, which contains rich annotations for 900 English verbs (Rashkin et al., 2016). Among other things, for each verb, the Connotation Frames Lexicon provides scores (ranging from -0.87 to 0.8 ) for the writer's perspective toward the verb's subject and object. In the example above for the verb attack, the lexicon lists the writer's perspective toward the subject "A," the attacker, as -0.6 (strongly negative) and the object "B" as 0.23 (weakly positive).

We extract all subject-verb-object tuples containing at least one target group label using the Spacy dependency parser ${ }^{3}$. For each subject and object, we capture the noun and the modifying adjectives, as group labels (such as gay) can often take either nominal or adjectival forms. For each tuple, we use the Connotation Frames lexicon to determine the writer's perspective toward the noun phrase containing the group label. We then average perspective scores over all tuples.

\subsubsection{Word Embedding Neighbor Valence}

While a Connotation Frames approach can be more precise than word-counting valence analysis, it limits us to analyzing SVO triples, which excludes a large portion of the available data about the target groups. This reveals a conundrum: broader context can provide valuable insights into the implicit evaluations of a social group, but we also want to directly probe attitudes toward the group itself.

We address this tension by training vector space models to represent the data, in which each unique word in a large corpus is represented by a vector (embedding) in high-dimensional space. The geometry of the resulting vector space captures many semantic relations between words. Furthermore, prior work has shown that vector space models trained on corpora from different time periods can capture semantic change (Kulkarni et al., 2015; Hamilton et al., 2016). For example, diachronic word embeddings reveal that the word gay meant "cheerful" or "dapper" in the early twentieth century, but shifted to its current meaning of sexual orientation by the 1970s. Because word embeddings are created from real-world data, they contain realworld biases. For example, Bolukbasi et al. (2016) demonstrated that gender stereotypes are deeply ingrained in these systems. 
Though problematic for the widespread use of these models in computational systems, these revealed biases indicate that word embeddings can actually be used to identify stereotypes about social groups and understand how they change over time (Garg et al., 2018).

This technique can similarly be applied to understand how a social group is negatively evaluated within a large text corpus. If the vector corresponding to a social group label is located in the semantic embedding space near words with clearly negative evaluations, that group is likely negatively evaluated (and possibly dehumanized) in the text.

We first preprocess the data by lowercasing, removing numbers, and removing punctuation. We then use the word2vec skip-gram model to create word embeddings (Mikolov et al., 2013). We use Gensim's default parameters with two exceptions; we train our models for ten iterations in order to ensure that the models converge to the optimal weights and we set the window size to 10 words, as word vectors trained with larger window sizes tend to capture more semantic relationships between words (Levy and Goldberg, 2014) ${ }^{4}$. For our diachronic analysis, we first train word2vec on the entire corpus, and then use the resulting vectors to initialize word2vec models for each year of data in order to encourage coherence and stability across years. After training word2vec, we zero-center and normalize all embeddings to alleviate the hubness problem (Dinu et al., 2014).

We then identify vectors for group labels by taking the centroid of all morphological forms of the label, weighted by frequency. For example, the vector representation for the label gay is actually the weighted centroid of the words gay and gays. This enables us to simultaneously account for adjectival, singular nominal, and plural nominal forms for each social group label with a single vector. Finally, we estimate the valence for each group label by identifying its 500 nearest neighbors via cosine similarity, and calculating the average valence of all neighbors that appear in the NRC VAD Valence Lexicon ${ }^{5}$.

We also induce a valence score directly from a group label's vector representation by adapting the regression-based sentiment prediction from Field and Tsvetkov (2019) for word embeddings. This approach yielded similar results as analyzing nearest neighbor valence but was difficult to interpret. More details for and results from this technique can be found in the Supplementary Material.

\subsection{Denial of Agency}

Denial of agency refers to the lack of attributing a target group member with the ability to control their own actions or decisions (Tipler and Ruscher, 2014). Automatically detecting the extent to which a writer attributes cognitive abilities to a target group member is an extraordinarily challenging computational task. Fortunately, the same lexicons used to operationalize negative

\footnotetext{
${ }^{4}$ https://radimrehurek.com/gensim/models/word2vec.html

${ }^{5} \mathrm{We}$ conducted additional analyses by considering $25,50,100,250$, and 1,000 nearest neighbors, which yielded similar results and can be found in the Supplementary Material.
}

evaluations provide resources for measuring lexical signals of denial of agency.

\subsubsection{Connotation Frames}

As in section 3.1, we use Connotation Frames to quantify the amount of agency attributed to a target group. We use Sap et al.'s extension of Connotation Frames for agency (Sap et al., 2017). Following Sap et al.'s interpretation, entities with high agency exert a high degree of control over their own decisions and are active decision-makers, while entities with low agency are more passive (Sap et al., 2017). This contrast is particularly apparent in example sentences, such as $X$ searched for $Y$ and $X$ waited for $Y$, where the verb searched gives $\mathrm{X}$ high agency and waited gives X low agency (Sap et al., 2017). Additionally, Sap et al.'s released lexicon for agency indicates that subjects of verbs such as attack and praise have high agency, while subjects of doubts and needs have low agency (Sap et al., 2017).

This lexicon considers the agency attributed to subjects of nearly 2,000 transitive and intransitive verbs. To use this lexicon to quantify denial of agency, we extract all sentences' head verbs and their subjects, where the subject noun phrase contains a target group label. Unlike Rashkin et al.'s real-valued Connotation Frames lexicon for perspective, the agency lexicon only provides binary labels, so we calculate the fraction of subject-verb pairs where the subject has high agency.

\subsubsection{Word Embedding Neighbor Dominance}

The NRC VAD Dominance Lexicon provides another resource for quantifying dehumanization (Mohammad, 2018). The NRC VAD lexicon's dominance dimension contains real-valued scores between zero and one for 20,000 English words. However, the dominance lexicon primarily captures power, which is distinct from but closely related to agency. While power refers to one's control over others, agency refers to one's control over oneself. While this lexicon is a proxy, it qualitatively appears to capture signals of denial of agency; the highest dominance words are powerful, leadership, success, and govern, while the lowest dominance words are weak, frail, empty, and penniless. We thus take the same approach as in section 3.1.3, but instead calculate the average dominance of the 500 nearest neighbors to each group label representation ${ }^{5}$.

As in section 3.1.3, we also induced a dominance score directly from a group label's vector representation by adapting the regression-based sentiment prediction from Field and Tsvetkov (2019) for word embeddings. More details and results for this technique can be found in the Supplementary Material.

\subsection{Moral Disgust}

To operationalize moral disgust with lexical techniques, we draw inspiration from Moral Foundations theory, which postulates that there are five dimensions of moral intuitions: care, fairness/proportionality, loyalty/ingroup, authority/respect, and sanctity/purity (Haidt and Graham, 2007). The negative end of the sanctity/purity dimension corresponds to moral disgust. While we do not directly 
incorporate Moral Foundations Theory in our framework for dehumanization, we utilize lexicons created by Graham et al. (2009) corresponding to each moral foundation. The dictionary for moral disgust includes over thirty words and stems, including disgust ${ }^{*}$, sin, pervert, and obscen ${ }^{*}$ (the asterisks indicate that the dictionary includes all words containing the preceding prefix $)^{6}$.

We opt for a vector approach instead of counting raw frequencies of moral disgust-related words because such words are rare in our news corpus. Furthermore, vectors capture associations with the group label itself, while word counts would not directly capture such associations. Using the word embeddings from section 3.1.3, we construct a vector to represent the concept of moral disgust by averaging the vectors for all words in the "Moral Disgust" dictionary, weighted by frequency. This method of creating a vector from the Moral Foundations dictionary resembles that used by Garten et al. (2016). We identify implicit associations between a social group and moral disgust by calculating cosine similarity between the group label's vector and the Moral Disgust concept vector, where a higher similarity suggests closer associations between the social group and moral disgust.

\subsection{Vermin as a Dehumanizing Metaphor}

Metaphors comparing humans to vermin have been especially prominent in dehumanizing groups throughout history (Haslam, 2006; Steuter and Wills, 2010). Even if a marginalized social group is not directly equated to vermin in the press, this metaphor may be invoked in more subtle ways, such as through the use of verbs that are also associated with vermin (like scurry as opposed to the more neutral hurry) (Marshall and Shapiro, 2018). While there is some natural language processing work on the complex task of metaphor detection (e.g., Tsvetkov et al., 2014), these systems cannot easily quantify such indirect associations.

We thus quantify the metaphorical relationship between a social group and vermin by calculating similarities between these concepts in a distributional semantic vector space. As with moral disgust, we create a Vermin concept vector by averaging the following vermin words' vectors, weighted by frequency: vermin, rodent(s), rat(s) mice, cockroaches, termite(s), bedbug(s), fleas ${ }^{7}$. We do not include the singular mouse or flea because non-vermin senses of those words were more frequent, and word2vec does not account for polysemy. We calculate cosine similarity between each group label and the Vermin concept vector, where a high cosine similarity suggests that the group is closely associated with vermin.

Table 1 provides an overview of the four elements of dehumanization that we study and the lexical techniques used to quantify them.

\section{DATA}

The data for our case study spans over 30 years of articles from the New York Times, from January 1986 to December 2015,

\footnotetext{
${ }^{6}$ https://www.moralfoundations.org/othermaterials

${ }^{7}$ Largely inspired by https://en.wikipedia.org/wiki/Vermin
}

TABLE 1 | Overview of linguistic correlates and our operationalizations for four elements of dehumanization.

\begin{tabular}{ll}
\hline $\begin{array}{l}\text { Dehumanization } \\
\text { element }\end{array}$ & Operationalization \\
\hline $\begin{array}{l}\text { Negative } \\
\text { evaluation of }\end{array}$ & $\begin{array}{l}\text { Paragraph-level sentiment analysis } \\
\text { target group }\end{array}$ \\
\hline Denial of agency & Word embedding neighbor valence \\
\hline Moral disgust & Connotation frames of agency \\
\hline Vermin metaphor & Word embedding neighbor dominance \\
\hline
\end{tabular}

and was originally collected by Fast and Horvitz (2016). The articles come from all sections of the newspaper, such as "World," "New York \& Region," "Opinion," "Style," and "Sports." Our distributional semantic methods rely on all of the available data in order to obtain the most fine-grained understanding of the relationships between words possible. For the other techniques, we extract paragraphs containing any of the following words from a predetermined list of LGTBQ terms: gay(s), lesbian(s), bisexual(s), homosexual(s), transgender(s), transsexual(s), transexual(s), transvestite(s), transgendered, asexual, agender, aromantic, lgb, lgbt, lgbtq, lgbtqia, glbt, lgbtqqia, genderqueer, genderfluid, intersex, pansexual.

Each acronym label is matched insensitive to case and punctuation. Some currently prominent LGBTQ terms, such as queer and trans are not included in this study, as other senses of these words were more frequent in earlier years. We filter out paragraphs from sections that typically do not pertain to news, such as "Arts," "Theater," and "Movies." While these sections could provide valuable information, we focus on representation of LGBTQ groups in more news-related contexts.

A challenging question when analyzing mass media for subjective attitudes is deciding whose perspective we want to capture: an individual reporter, the institution, or society at large? In this case study, we aim to identify the institution's dehumanizing attitudes toward LGBTQ people. We represent the New York Times institution as a combination of the journalists' words in news articles, direct quotes, paraphrases from interviews, and published opinion articles. Therefore, despite our news focus, we include data from "Opinion" sections; while opinion articles are stylistically different from traditional journalistic reporting due to more overt biases and arguments, these articles are important in constructing the institution's perspective. In addition, we consider all text in each relevant paragraph, including quotes and paraphrases, because they are important to a newspaper's framing of an issue, as particular quotes representing specific stances are intentionally included or excluded from any given article (Niculae et al., 2015).

We refer to the remaining subset of the New York Times data after filtering as the LGBTQ corpus. The LGBTQ corpus consists of 93,977 paragraphs and 7.36 million tokens. A large increase in reporting on LGBTQ-related issues has led to a skewed distribution in the amount of data over years, with 


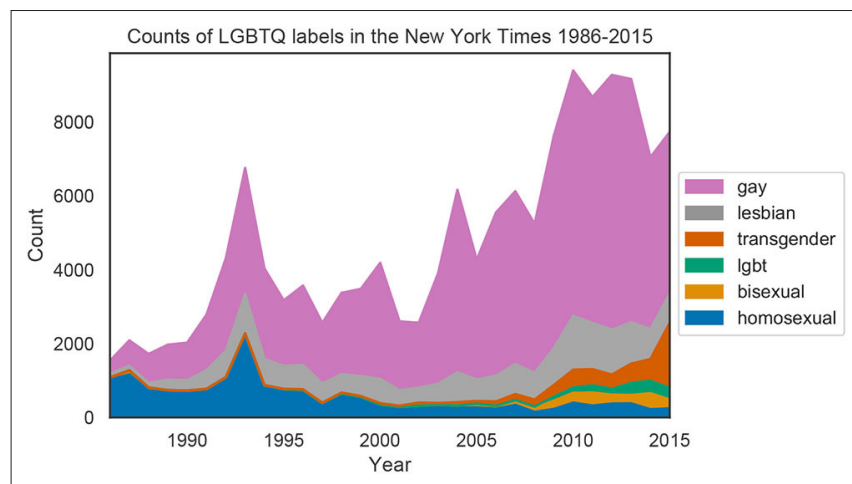

FIGURE 1 | Counts for the six most frequent LGBTQ labels in each year of the New York Times data.

1986 containing the least data (1,144 paragraphs and 73,549 tokens) and 2012 containing the most (5,924 paragraphs and 465,254 tokens).

For all experiments, we also include results for the terms American and Americans. We include American(s) to contrast changes in LGBTQ labels' representation with another social group label. This ensures that the changes we find in dehumanizing language toward LGBTQ groups do not apply uniformly to all social groups, and are thus not merely an artifact of the publication's overall language change. While a natural "control" variable would be labels, such as straight or heterosexual, these terms only occurred within discussions of LGBTQ communities because they name socially unmarked categories. We also considered comparing LGBTQ labels to person/people, but because word embedding-based experiments are sensitive to syntactic forms, we opt for a label that behaves more syntactically similar to gay and homosexual, particularly with both nominal and adjectival uses. Nevertheless, American(s) is by no means a neutral control variable. Because of its ingroup status for the New York Times (a U.S. institution), we expect our measurements to show that American(s) appears in more humanizing contexts than LGBTQ labels; however, we do not expect to find substantial changes in the use of American(s) over time.

Figure 1 shows the counts of group labels for each year in the New York Times from 1986 to 2015. For visualization purposes, only words with a total count $>1,000$ are shown. The relative frequency of homosexual decreased substantially over time, while gay, lesbian, and bisexual are more frequent in later years. The terms lgbt and transgender also emerged after 2000. Counts for all LGBTQ labels can be found in the Supplementary Material.

\section{RESULTS}

\subsection{Word Embeddings}

Using all of the New York Times data, we create word2vec models for each year using the methods described in section 3.1.3. Because our computational techniques rely upon these word2vec models, it is useful to gain a sense of how LGBTQ terms are semantically represented within these models. We thus
TABLE 2 | Nearest words to weighted average of all LGBTQ terms' vectors in 1986, 2000, and 2015.

\begin{tabular}{lll}
\hline $\mathbf{1 9 8 6}$ & $\mathbf{2 0 0 0}$ & $\mathbf{2 0 1 5}$ \\
\hline Sex & Interracial & Sex \\
Premarital & Openly & Non- \\
& & transgender \\
Sexual & Unwed & Unmarried \\
Abortion & Homophobia & Interracial \\
Promiscuity & Premarital & Closeted \\
Polygamy & Ordination & Equality \\
Promiscuous & Non-whites & Couples \\
Vigilantism & Ordaining & Abortion \\
Bestiality & Discrimination & Sexuality \\
Pornography & Abortion & Antiabortion \\
\hline
\end{tabular}

inspect the ten nearest neighbors, or most similar words, to LGBTQ terms in different years. Note that the neighboring words in Tables 2, 3 are shown purely for qualitative investigation; our measures for quantifying each dehumanization component incorporate far more information from the word2vec models beyond the top ten neighbors.

Table 2 shows the 10 nearest neighbors (by cosine similarity) to our vector representation of all LGBTQ terms, which is the weighted average of the embeddings of all LGBTQ terms considered. For visual convenience, we filter out words occurring fewer than ten times, proper names, as well as other LGBTQ labels and forms of the word heterosexual, which are common neighbors for all terms across all years.

Table 2 shows that in 1986, LGBTQ groups were most highly associated with words that often convey a sense of sexual deviancy, including promiscuity, promiscuous, polygamy, bestiality, and pornography. These associations suggest that LGBTQ people were dehumanized to some extent at this time, and their identities were not fully recognized or valued. This shifted by 2000 , where we no longer see associations between LGBTQ groups and ideas that evoke moral disgust. Instead, the 2000 vector space shows that LGBTQ people have become more associated with civil rights issues (suggested by interracial, homophobia, and discrimination). The words ordination and ordaining likely appear due to major controversies that arose at this time about whether LGBTQ people should be permitted to be ordained. We also see some indications of self-identification with the term openly. Finally, we see a slight shift toward associations with identity in 2015, with nearby words including nontransgender, closeted, equality, and sexuality. Curiously, the word abortion is a nearby term for all 3 years. Perhaps this is because opinions toward abortion and LGBTQ rights seem to be divided along similar partisan lines.

Table 3 shows the ten nearest neighboring words to our representations of gay and homosexual after filtering out proper names, words appearing $<10$ times that year, other LGBTQ terms, and forms of heterosexual. Table 3 reveals variation in social meaning between gay and homosexual despite denotational similarity, and these differences intensify over time. In 1986, 
TABLE 3 | Nearest words to vector representations of gay and homosexual in 1986, 2000, and 2015.

\begin{tabular}{|c|c|c|c|c|c|}
\hline \multicolumn{2}{|c|}{1986} & \multicolumn{2}{|c|}{2000} & \multicolumn{2}{|c|}{2015} \\
\hline Gay & Homosexual & Gay & Homosexual & Gay & Homosexual \\
\hline Homophobia & Premarital & Interracial & Premarital & Interracial & Premarital \\
\hline Women & Abortion & Openly & Openly & Sex & Sexual \\
\hline Feminist & Sexual & Homophobia & Deviant & Couples & Bestiality \\
\hline Vigilante & Sex & Unwed & Interracial & Mormons & Pedophilia \\
\hline Vigilantism & Promiscuity & Ordination & Promiscuity & Marriage & Adultery \\
\hline Suffrage & Polygamy & Premarital & Immoral & Closeted & Infanticide \\
\hline Sexism & Anal & Abortion & Sexual & Equality & Abhorrent \\
\hline A.c.l.u. & Intercourse & Antigay & Criminalizing & Abortion & Sex \\
\hline Amen & Consenting & Discrimination & Polygamy & Unmarried & Feticide \\
\hline Queer & Consensual & Marriagelike & Consensual & Openly & Fornication \\
\hline
\end{tabular}

gay is associated with terms of discrimination, civil rights and activism, such as homophobia, feminist, suffrage, sexism, and a.c.l.u. On the other hand, homosexual is primarily associated with words related to sexual activity (e.g., promiscuity, anal, intercourse, consenting).

In 1986, this pattern may be due to discussions about sexual transmission of AIDS, but the pejoration of homosexual continues over time. While gay becomes associated with issues related to marriage equality and identity in 2015, homosexual becomes extremely associated with moral disgust and illicit activity, with nearest neighbors including bestiality, pedophilia, adultery, infanticide, and abhorrent.

This qualitative analysis of word embedding neighbors reveals significant variation and change in the social meanings associated with LGBTQ group labels, with clear relationships to dehumanizing language. We will now present our quantitative results for measuring each component of dehumanization.

\subsection{Negative Evaluation Toward Target Group}

\subsubsection{Quantitative Results}

\subsubsection{Paragraph-level valence analysis}

Figure 2A shows the average valence for paragraphs containing LGBTQ labels [and American(s) for comparison], where a paragraph's valence is simply the average valence over its words (or lemmas) that appear in the NRC VAD Valence Lexicon. The NRC VAD lexicons actually contain several LGBTQ terms, which all have lower than the average valence score of 0.5: transsexual (0.264), homosexual (0.333), lesbian (0.385), gay (0.388), and bisexual (0.438). These values contrast starkly with more positively-valenced entries in the lexicon, such as heterosexual (0.561), person (0.646), human (0.767), man (0.688), and woman (0.865). These disparities likely reveal biases among the human annotators whose judgments were used to construct the NRC VAD lexicon (Mohammad, 2018). While the lexicon may itself be an interesting artifact of dehumanizing attitudes toward LGBTQ people, we remove these terms before calculating paragraphlevel valence scores in order to isolate linguistic signals in the New York Times data from annotation biases. Without this preprocessing step, the temporal trends and relative differences between all LGBTQ terms, gay, and homosexual remain roughly the same, but all LGBTQ labels occur in significantly more negative paragraphs than American.

Figure 2A shows the average paragraph valence. For visualization purposes, we present the results over 5-year intervals due to data sparsity in later years for homosexual (there were just 208 paragraphs containing homosexual in 2014, relative to 3,669 containing gay in the same year). Analysis of overlapping confidence intervals and Wilcoxon signed-rank tests over the means for each of the 30 years indicates that gay and all LGBTQ terms occur in significantly more positive paragraphs than homosexual ( $p<0.0001)$. A linear regression analysis over all years reveals that all LGBTQ terms, gay, and homosexual all significantly increase in paragraph-level valence over time $(p<0.0001)$. However, when considering just the last 15 years, gay still significantly increases in paragraph-level valence, while homosexual may be trending downward, although this trend does not reach significance in our data $(p=0.078)$.

The paragraph-level valence analysis shown in Figure 2A suggests that LGBTQ groups have become increasingly positively evaluated over time, and thus likely less dehumanized in the New York Times. However, the slight downward trend in valence for paragraphs containing homosexual between 2001 and 2015 suggests that evaluations of people described as homosexual have not improved in the same way as those described by other labels.

Finally, this measurement does not support our initial hypothesis that LGBTQ groups have been more negatively evaluated than American(s), but still reveals that the observed trends for LGBTQ labels are not merely artifacts of changing reporting styles, since paragraphs containing American(s) show a very different pattern. Overall, this result demonstrates substantial language change in the New York Times's discussion of LGBTQ people as well as variation in the contexts where different group labels appear, particularly homosexual.

\subsubsection{Connotation frames of perspective}

Figure 2B shows the writer's average perspective (valence) toward noun phrases containing either any LGBTQ labels, gay (s), homosexual(s), or the comparison group American(s) using the 

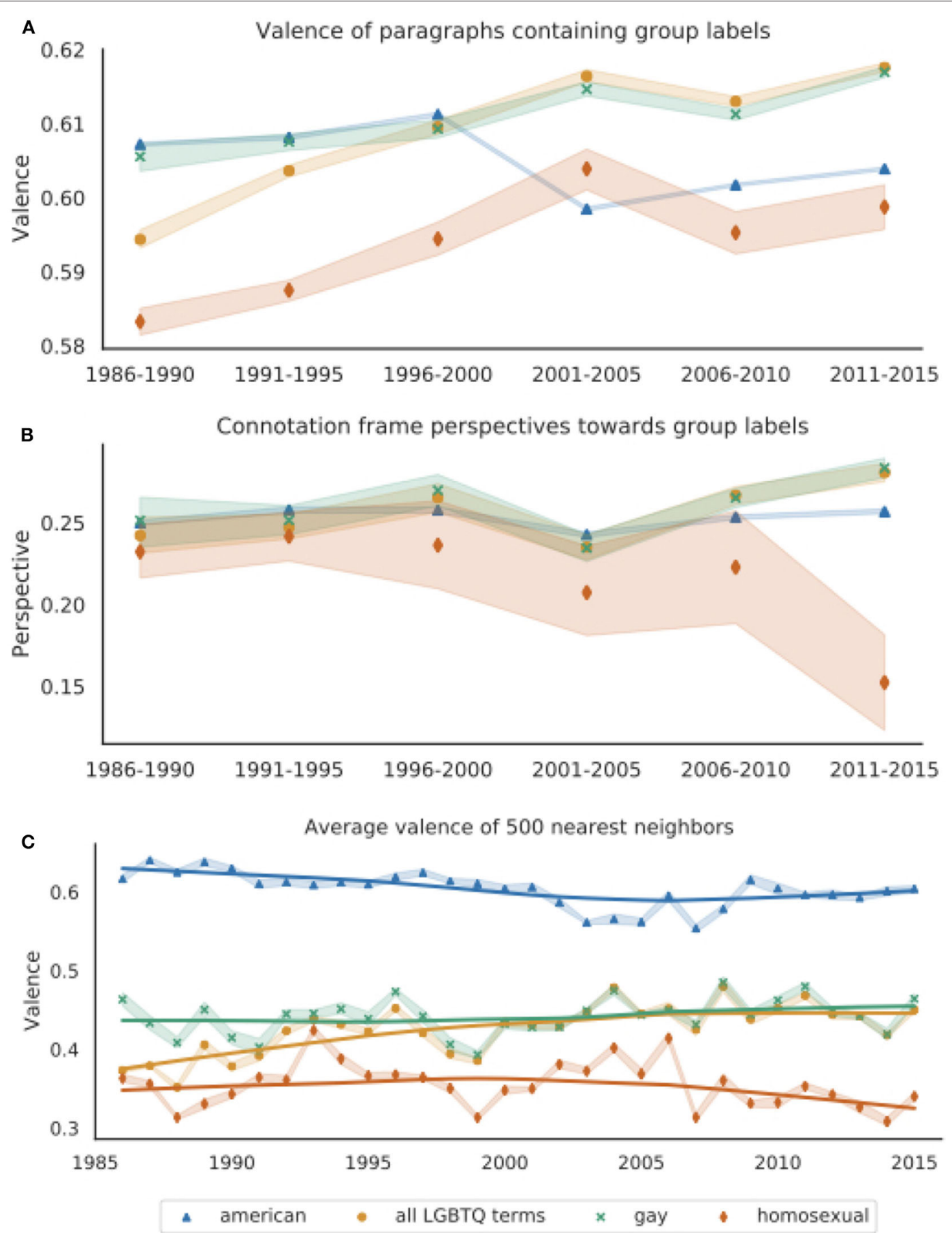

FIGURE 2 | (A) Average paragraph-level valence for paragraphs containing gay, homosexual, any LGBTQ term, and American, grouped into 5-years intervals. Paragraph-level scores are calculated as the average valence over all words that appear in the NRC VAD Valence Lexicon, which range from 0 (most negative) to 1 (most positive) (Mohammad, 2018). Paragraphs containing LGBTQ labels become more positive over time. Paragraphs containing homosexual are significantly more negative than those containing other LGBTQ labels. (B) Average connotation frame perspective scores over 5-years intervals. Scores are calculated for each subject-verb-object triple containing these group labels as the writer's perspective based on the head verb's entry in the Connotation Frames lexicon (Rashkin et al., 2016). (C) Average valence of 500 nearest words to vector representations of gay, homosexual, all LGBTQ terms, and American, averaged over 10 word2vec models trained on New York Times data from each year. The solid lines are Lowess curves for visualization purposes. Words' valence scores are from the NRC VAD Valence Lexicon. For all plots, the shaded bands represent 95\% confidence intervals. 
Connotation Frames lexicon (Rashkin et al., 2016). The wide variation, particularly for homosexual, is likely due to sparsity, as limiting the connotation frames analysis to verbs' immediate subject and direct object noun phrase dependents (consisting of only determiners, adjectives, and nouns) greatly reduced the amount of data for each year; there were only 39 triples for homosexual in 2015. We thus show results aggregated over 5years intervals.

As with paragraph-level valence, the writer's perspective toward the label homosexual is significantly more negative than toward gay $(p<0.001)$. Linear regression indicates that perspectives toward noun phrases named by any LGBTQ term, gay, and American have all significantly increased over time $(p<$ $0.01)$. However, the trends are still quite different, as the slopes for gay and all LGBTQ terms are an order of magnitude greater than American $\left[m=(1.1 \pm 0.39) \times 10^{-4}\right.$ for American, $m=(1.4 \pm$ $0.18) \times 10^{-3}$ for all LGBTQ terms, and $m=(1.1 \pm 0.22) \times 10^{-3}$ for gay]. Furthermore, the writer's perspective toward noun phrases containing homosexual have significantly decreased over time $(p<0.0001)$.

Overall, Connotation Frames' perspective scores reveal a similar pattern as the paragraph-level valence analysis, where LGBTQ groups overall appear to be more positively evaluated in the New York Times over time. Unlike gay and the aggregated all LGBTQ terms, the label homosexual undergoes pejoration, as homosexual becomes increasingly used when (implicitly) expressing negative attitudes toward LGBTQ people.

\subsubsection{Word embedding neighbor valence}

Figure 2C shows the average valence scores of the 500 nearest neighbors to the vector representations of gay, homosexual, all LGBTQ terms, and American for each year. In contrast to our other techniques to quantify negative evaluations of a target group, this measurement notably shows that the valence of American's neighboring words is significantly greater than any of the LGBTQ group representations' neighbors every year (Wilcoxon's signed-rank test, $p<0.0001$ ), indicating that American is used in more positive contexts than LGBTQ terms. Furthermore, all LGBTQ vectors' neighbors have an average valence below the neutral 0.5 . The average valence for neighboring words of gay and the aggregated all LGBTQ terms representation significantly increase over time $(p<0.0001)$, suggesting some increasing humanization in the language used in discussions of LGBTQ people.

Figure 2C also reveals dramatic connotational differences between gay and homosexual. As shown by non-overlapping confidence intervals and a Wilcoxon signed-rank test, the average valence for homosexual's neighbors is significantly lower than gay's neighbors over all years $(p<0.0001)$. Furthermore, while gay's average neighbor valence increases over time $(p<0.0001)$, homosexual's neighboring words become slightly but significantly more negative over time $(p<0.001)$. Analyzing the valence of the nearest neighbors indicates that homosexual has long been used in more negative (and potentially dehumanizing) contexts than gay, and that these words' meanings have further diverged as the label homosexual has been used in increasingly negative contexts over time.

\subsubsection{Qualitative Analysis}

\subsubsection{Paragraph-level valence analysis}

How well does paragraph-level valence analysis capture negative evaluations of a target group? To facilitate a qualitative evaluation of this technique, we identify several hundred paragraphs with the highest and lowest average valence. Most paragraphs with high valence scores appear to express positive evaluations of LGBTQ individuals, and those with low scores express negative evaluations.

Table 4 contain examples with extremely high and low valence. We identify several major themes from these results. Most paragraphs with high valence scores emphasize equal rights, while some focus on the activities of advocacy organizations. On the other end, paragraphs with extremely low valence often focus on violence against LGBTQ people, disease (especially AIDS), and LGBTQ issues internationally. Other themes that emerge in low-valence paragraphs include reports on (and direct quotes from) public figures who dehumanized LGBTQ people and portrayals of LGBTQ people as reckless, irresponsible, and angry.

While this technique accurately captures the valence for many paragraphs, we also identify several shortcomings. Some extreme outliers are extremely short paragraphs, including subtitles within articles which are included as paragraphs in the data. Table 5 shows several examples that were mischaracterized by our paragraph-level valence analysis technique. In addition, there are several paragraphs with highly positive average valence that actually express negative evaluations of LGBTQ people. The valence of the third paragraph in Table 5 is skewed by the positive words supported and marriage even though the paragraph is actually discussing low support for gay marriage. While the fourth paragraph argues that gay couples would be subpar parents relative to straight couples, it uses positive terms, such as love and ideal. Furthermore, kinship terms tend to be assigned highly positive values in the NRC VAD Valence Lexicon, including child and family. Similarly, even though the final example describes discrimination based on sexual orientation, the paragraph's average valence is impacted by positive kinship terms, such as father $(0.812)$ and mother $(0.931)^{8}$.

Overall, our qualitative analysis shows that highly positive valence often accompanies expressions of positive evaluation toward LGBTQ groups, and low valence often accompanies expressions of negative evaluation. However, paragraph-level valence scores are also impacted by specific words cued by various topics; paragraphs about same-sex marriage tend to be more positive because words like marriage, marry, and couple have high valence scores while paragraphs reporting on hate crimes tend to be more negative because they contain low-valence words related to crime, violence, and injury. Furthermore, this method cannot disentangle perspectives within the text; although there are linguistic signals of dehumanization expressed in reports on anti-LGBTQ violence and homophobic speech, these dehumanizing attitudes are not necessarily from the viewpoint of the journalist or the institution. Nevertheless, there

\footnotetext{
${ }^{8} \mathrm{We}$ also conducted paragraph-level sentiment analysis using binary positive vs. negative emotion lexicons, such as LIWC (Pennebaker et al., 2001), but found similar quantitative results and no qualitative improvement over the VAD lexicon.
} 
TABLE 4 | Example paragraphs with extremely high and low valence scores, along with an interpretation of the patterns we find.

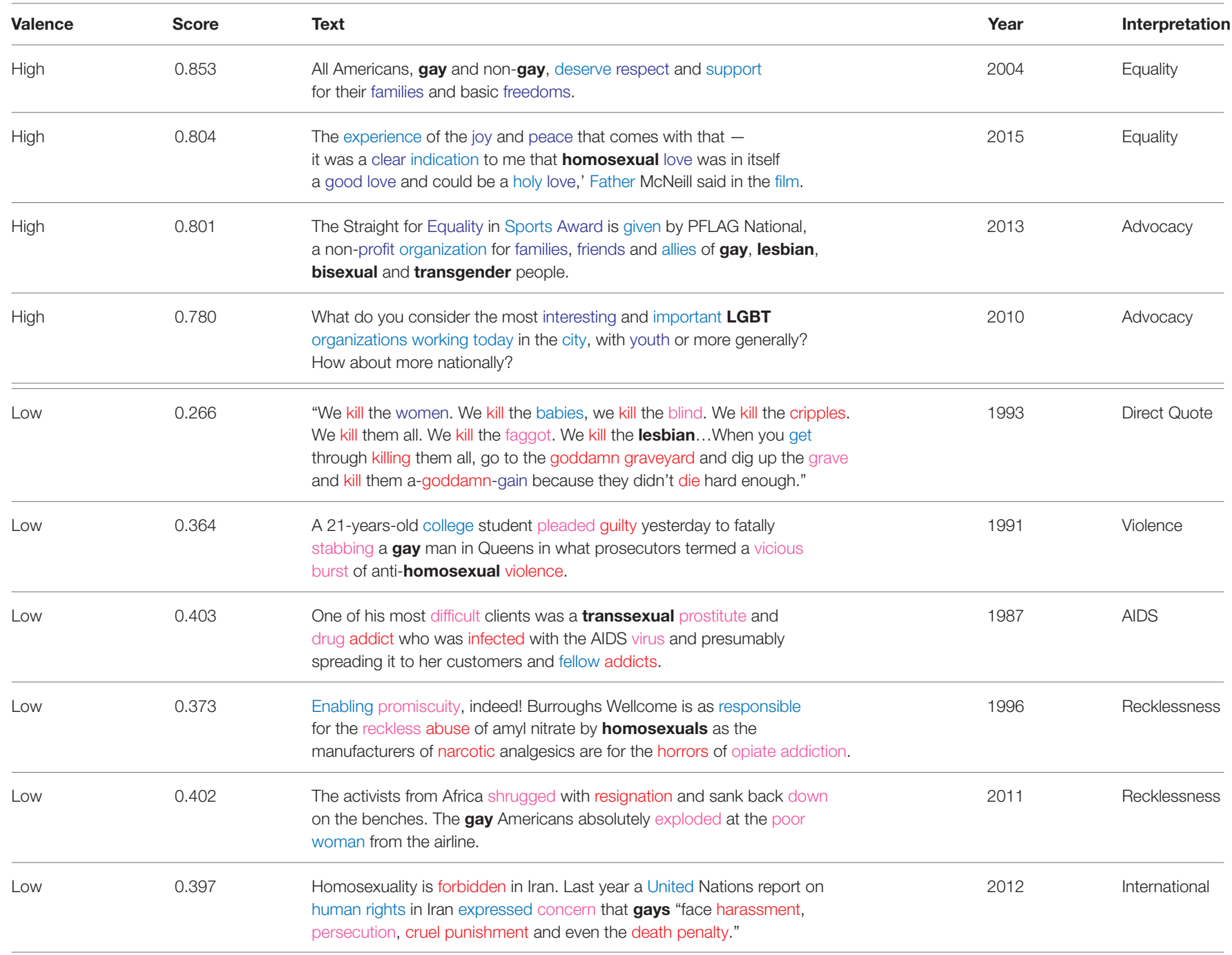

Words with extremely high valence scores (>0.85) appear in blue, and somewhat high-valence words (scores between 0.7 and 0.85 ) appear in light blue. Words with extremely low valence scores (<0.15) appear in red, and somewhat low-valence words (scores between 0.15 and 0.3 ) appear in pink. LGBTQ terms are shown in bold.

TABLE 5 | Examples mischaracterized by paragraph-level valence analysis.

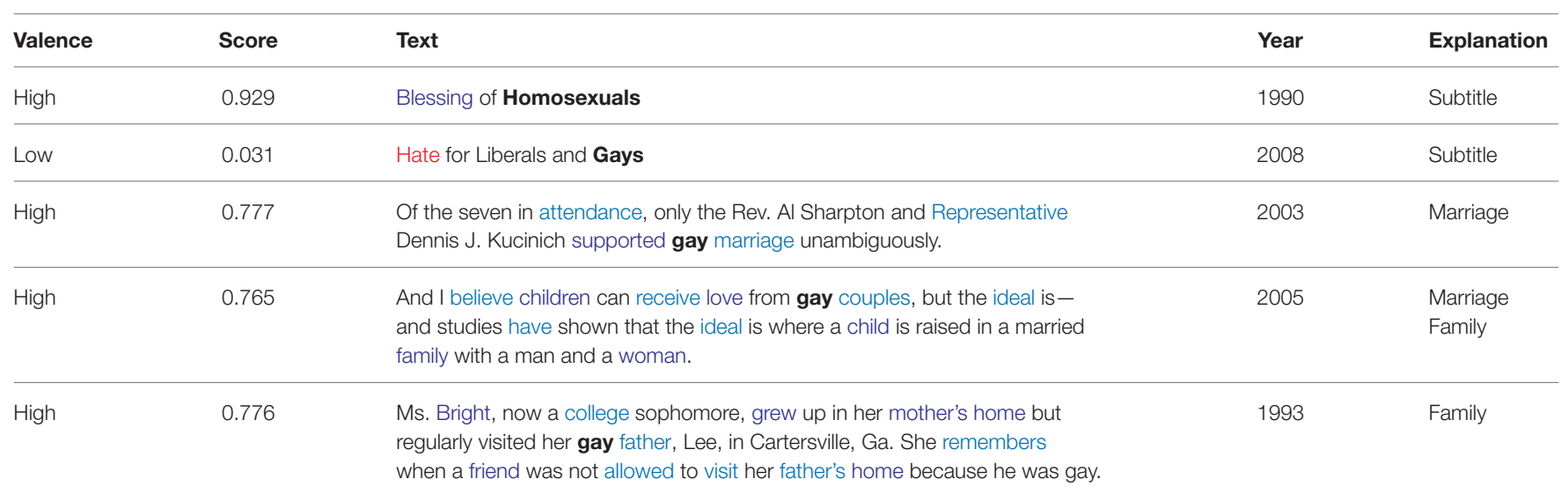

Words with extremely high valence scores (>0.85) appear in blue, and somewhat high-valence words (scores between 0.7 and 0.85 ) appear in light blue. Words with extremely low valence scores $(<0.15)$ appear in red. LGBTQ terms are shown in bold. 
TABLE 6 | Examples of paragraphs where the writer expresses highly positive and negative perspective toward LGBTQ groups, according to the Connotation Frames lexicon.

\begin{tabular}{|c|c|c|c|c|}
\hline Perspective & Score & Text & svo & Year \\
\hline Negative & -0.83 & $\begin{array}{l}\text { The most forceful comment came from Cardinal Anthony J. Bevilacqua of } \\
\text { Philadelphia, who said his archdiocese screened out gay candidates. "We feel a } \\
\text { person who is homosexual-oriented is not a suitable candidate for the } \\
\text { priesthood, even if he had never committed any homosexual act," the } \\
\text { cardinal said. }\end{array}$ & $\begin{array}{l}\text { S: he } \\
\text { V: committed } \\
\text { O: any } \\
\text { homosexual act }\end{array}$ & 2002 \\
\hline Positive & +0.80 & $\begin{array}{l}\text { "Gays are accepted here and respected here," said Mayor Tony Tarracino. } \\
\text { "The gays saved a lot of the oldest parts of town, and they brought in } \\
\text { art and culture. They deserve a lot of credit for what Key West is today." }\end{array}$ & $\begin{array}{l}\text { S: the gays } \\
\text { V: saved } \\
\text { O: a lot }\end{array}$ & 1990 \\
\hline Positive & +0.80 & $\begin{array}{l}\text { In his speech, he praised gay rights advocates for their hard work and } \\
\text { also thanked many elected officials, including his predecessor, } \\
\text { Gov. David A. Paterson, and the four Republican state senators who } \\
\text { provided the critical votes to pass the marriage bill and whom Mr. Cuomo } \\
\text { named one by one to some of the loudest applause of the evening. }\end{array}$ & $\begin{array}{l}\text { S: he } \\
\text { V: praised } \\
\text { O: gay rights } \\
\text { advocates }\end{array}$ & 2011 \\
\hline
\end{tabular}

\section{Assigned perspective}

\begin{tabular}{|c|c|c|c|c|}
\hline Negative & -0.87 & $\begin{array}{l}\text { Previously, Judge Vaughn Walker, who ruled the ban against } \\
\text { same-sex unions unconstitutional in federal court, had said that } \\
\text { ProtectMarriage could not appeal his decision to the Ninth Circuit, } \\
\text { because they were never able to prove that gay marriage harmed them } \\
\text { in any way. }\end{array}$ & $\begin{array}{l}\text { S: gay marriage } \\
\text { V: harmed } \\
\text { O: them }\end{array}$ & 2011 \\
\hline Positive & +0.73 & $\begin{array}{l}\text { Following are excerpts from opinions by the Supreme Court today in } \\
\text { its decision that the Constitution does not protect private homosexual } \\
\text { relations between consenting adults (...) Justice Stevens wrote a } \\
\text { separate dissenting opinion, joined by Justices Brennan and Marshall. }\end{array}$ & $\begin{array}{l}\text { S: the } \\
\text { Constitution } \\
\text { V: protect } \\
\text { O: private } \\
\text { homosexual } \\
\text { relations }\end{array}$ & 1986 \\
\hline Positive & +0.70 & $\begin{array}{l}\text { Do you know there is a Congressional candidate from Missouri who is } \\
\text { saying that allowing gays into the military could strengthen Al Qaeda? } \\
\text { l'm thinking, how exactly would that work? "They dance better than me, } \\
\text { and they know how to accessorize. I'm very, very angry. It's time for jihad." }\end{array}$ & $\begin{array}{l}\text { S: gays } \\
\text { V: strengthen } \\
\text { O: Al Qaeda }\end{array}$ & 2010 \\
\hline
\end{tabular}

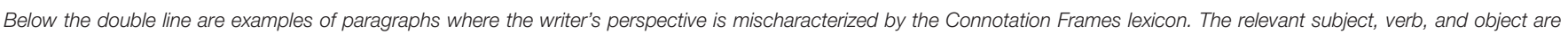
shown in bold.

could be an overall dehumanizing effect if the media's discussions of a marginalized social group emphasizes such events that harm people. Repeated associations between LGBTQ labels and such negative contexts could potentially contribute to negative evaluations of LGBTQ groups.

\subsubsection{Connotation frames of perspective}

To qualitatively analyze how well the connotation frames' lexicon capture negative evaluation of a target group, we identify SVO tuples where the verb indicates that the writer has extremely positive or negative perspective toward either the subject or object. The first paragraph in Table 6 contains an SVO tuple where the writer has the most negative perspective toward the noun phrases containing a group label. Inside a direct quote, this paragraph uses the phrase any homosexual act as the object to the verb committed, which has the effect of framing homosexuality as a crime. By deeming gay candidates unworthy of the priesthood, the speaker clearly negatively evaluates LGBTQ people. On the opposite end, many paragraphs labeled as containing extremely positive perspectives toward LGBTQ groups do appear to have positive evaluations of these groups. The second and third paragraphs of Table 6 illustrate this, where the gays are viewed positively for having saved a town, and gay rights advocates are praised for their work.

However, we found several instances where paragraphs are mislabeled, shown in the bottom half of Table 6. In the fourth paragraph of Table 6, our technique identifies gay marriage as the subject of the negative-perspective verb harmed, but does not account for the preceding text, which actually contradicts the premise that gay marriage causes harm, and thus does not overtly negatively evaluate of LGBTQ groups (although this particular example reveals the difficulty of operationalizing this component because ProtectMarriage groups strongly oppose same-sex marriage and may have negative evaluations of LGBTQ people). The second example similarly shows that this method does not adequately account for various forms of negation, as the positive-perspective verb protect is actually negated. The last example in Table 6 presents a complex case that is even 
challenging for qualitative analysis. Our method identifies gays as the subject of the verb strengthen, even though the subject should be the gerund allowing gays (into the military), and the lexicon's entry for the writer's perspective toward the subject of strengthen is a highly positive 0.7 . However, the object of this verb is the terrorist organization Al Qaeda; our background knowledge suggests that the capacity to strengthen Al Qaeda would reflect negative perspectives. However, this additional context provided by the rest of the paragraph indicates that the writer is being sarcastic and considers the proposition that gays have any impact on strengthening Al Qaeda to be ridiculous. Finally, the writer emphasizes their own stance in opposition to the Missouri congressional candidate by calling upon common stereotypes of gay people being good at dancing and accessorizing.

Measuring the connotation frames' lexicon perspective scores over verbs' subjects and direct objects cannot leverage as much context as measuring valence over paragraphs using the NRC VAD lexicon labeled for 20,000 words. However, this technique can make more fine-grained distinctions regarding the writer's (and institution's) attitudes directed toward LGBTQ people and is not as dramatically impacted by the emotional valence of the topic discussed. Neither technique can disentangle the journalist's perspective from those expressed by others and simply reported by the journalist. While removing direct quotations may partially address this issue, we deliberately do not remove text from direct quotes or paraphrases. The journalists and newspaper make intentional decisions about what text to include and exclude from quotations, which could still meaningfully represent their perspectives and values (Niculae et al., 2015).

\subsubsection{Word embedding neighbor valence}

Compared to the previous methods, one limitation of using word embeddings to quantify negative evaluations of a target group is that embeddings are not easily interpretable by analyzing a small sample of data. Instead, we assess this technique by identifying LGBTQ terms' nearest neighbors in several outlier years. To facilitate this qualitative analysis, we identify a set of unique nearest neighbors for each LGBTQ label in each outlier year, where a word is a unique nearest neighbor for a given LGBTQ term and year if it is not in that term's top 500 nearest neighbors in any other year.

Table 7 contains several example paragraphs that illustrate overarching themes for the outlier years 1993, 1999, and 2014. In 1999, gay, homosexual and the aggregated representation of all LGBTQ terms were all more closely associated with lowvalence words than in almost any other year. We connect this finding to a period of intense reporting in the months following the October 1998 murder of a gay Wyoming college student, Matthew Shepard, which drew national attention to anti-LGBTQ violence. Because LGBTQ labels frequently co-occurred with text about this incident, terms related to Matthew Shepard's case had closer representations to LGBTQ terms in this year. For example, gay and all LGBTQ terms's 500 nearest neighbors include wyoming in 1999 and shepard from the years 1998-2000. Unique nearest neighbors for gay in 1999 include other terms that could be connected to this incident, including homicidal, imprisoned, and hatred. Not only was Shepard's murder rooted in the dehumanization of LGBTQ people, but the media's emphasis on the gruesome details of Shepard's death further dehumanized him (Ott and Aoki, 2002). Ott and Aoki argue that the media's framing of this case actually further stigmatized LGBTQ people.

Our word embedding neighbor valence measure reveals that the most negative year for gay and all LGBTQ terms since 1999 was 2014, the second most-recent year of data. We identify several major themes in 2014 that co-occurred with LGBTQ group labels and possibly led to this distributional semantic pattern, primarily reporting on anti-LGBTQ laws and attitudes in Uganda and Russia (particularly in light of the 2014 Winter Olympics in Sochi). The terms athletes and winterolympics appeared in gay's nearest neighbors in 2014. In addition, the terms Uganda, Ugandan, and Mugisha (a Ugandan LGBT advocate) are among gay's unique nearest 500 neighbors in 2014.

Unlike in 1999 and 2014, LGBTQ terms in 1993 are associated with higher-valence words, especially homosexual. Homosexual's unique nearest neighbors in 1993 include the high-valence words pledge, civilian, readiness, and inclusion. These words are likely connected with numerous stories in 1993 covering the controversy over whether LGBTQ people should be allowed to serve in the military.

\subsection{Denial of Agency}

\subsubsection{Quantitative Results}

\subsubsection{Connotation frames of agency}

Figure 3A shows the agency of each group label based on its head verb's entry in the Connotation Frames lexicon for agency (Sap et al., 2017). As in Figure 2B, there is large variance due to data sparsity when using the Connotation Frames lexicon, particularly for homosexual, which is considerably less frequent than gay or other LGBTQ terms in later years. In order to maximize precision when extracting subject-verb pairs, we extract only nouns and their immediate adjectival modifiers, which limits the amount of data. We thus show average agency over 5-years intervals.

Wilcoxon signed-rank tests on the means for each group labels over all years indicate that gay occurs in contexts with significantly higher agency than homosexual $(p<0.0001)$. All four group labels significantly decrease in agency over time according to linear regressions over all 30 years $(p<0.001)$, but the slope for homosexual is much greater $[m=(-7.9 \pm 1.3) \times$ $10^{-3}$ for homosexual, compared to $m=(-3.9 \pm .55) \times 10^{-3}$ for gay, and $m=(-1.5 \pm .46) \times 10^{-3}$ for all LGBTQ terms]. Furthermore in the most recent 15 years, gay and all LGBTQ terms show no significant change $(p=0.097$ for gay and $p=0.14$ for all LGBTQ terms), but homosexual still decreases significantly in agency $(p<0.05)$.

Figure 3A suggests that LGBTQ groups experience greater denial of agency in the New York Times than the institution's in-group identifier American. Furthermore, people described as homosexual experience even more denial of agency than people who are described as gay. Unlike the improving attitudes indicated by our analysis of negative evaluations of a target group, it appears that denial of agency increased over time for all LGBTQ groups. However, the relatively rapid decrease in agency for homosexual is consistent with other results suggesting homosexual's pejoration. 
TABLE 7 | Example paragraphs from years where LGBTQ terms' nearest neighbors had exceptionally high and low valence.

\begin{tabular}{lll}
\hline Valence & Year & Example \\
\hline Low & 1999 & $\begin{array}{l}\text { Matthew Shepard, a gay college student in Wyoming, had been pistol-whipped and left to die after } \\
\text { being tied to a fence on Oct. 7, 1998. Aaron McKinney, who was charged with first-degree murder and } \\
\text { other crimes in connection with Mr. Shepard's killing, went on trial Monday, denying that the act was a } \\
\text { hate crime, but rather connected to drug use and outrage at a sexual advance he said Mr. Shepard made. }\end{array}$ \\
\hline Low & $\begin{array}{l}\text { Uganda's vehement anti-gay movement began in } 2009 \text { after a group of American preachers went to } \\
\text { Uganda for an anti-gay conference and then worked with Ugandan legislators to draft a bill that called } \\
\text { for putting gay people to death. While the bill was being debated, attacks against gay Ugandans began } \\
\text { to increase. In early 2011, David Kato, a slight, bespectacled man and one of the country's most } \\
\text { outspoken gay rights activists, was beaten to death with a hammer. }\end{array}$ \\
\hline Low & $\begin{array}{l}\text { "Hey, @McDonalds: You're sending \#CheersToSochi while goons wearing Olympic uniforms } \\
\text { assault LGBT people," read one comment last week, from the author and activist Dan Savage. }\end{array}$ \\
\hline High & $\begin{array}{l}\text { The regulations, which are to take effect Feb. 5, codify the Administration's policy that was worked } \\
\text { out as a compromise with the Joints Chiefs of Staff, who had defended the 50-years-old ban, arguing that } \\
\text { allowing homosexuals to serve openly would hurt the morale of troops, and thus hurt military readiness. }\end{array}$ \\
\hline
\end{tabular}

LGBTQ terms are shown in bold.
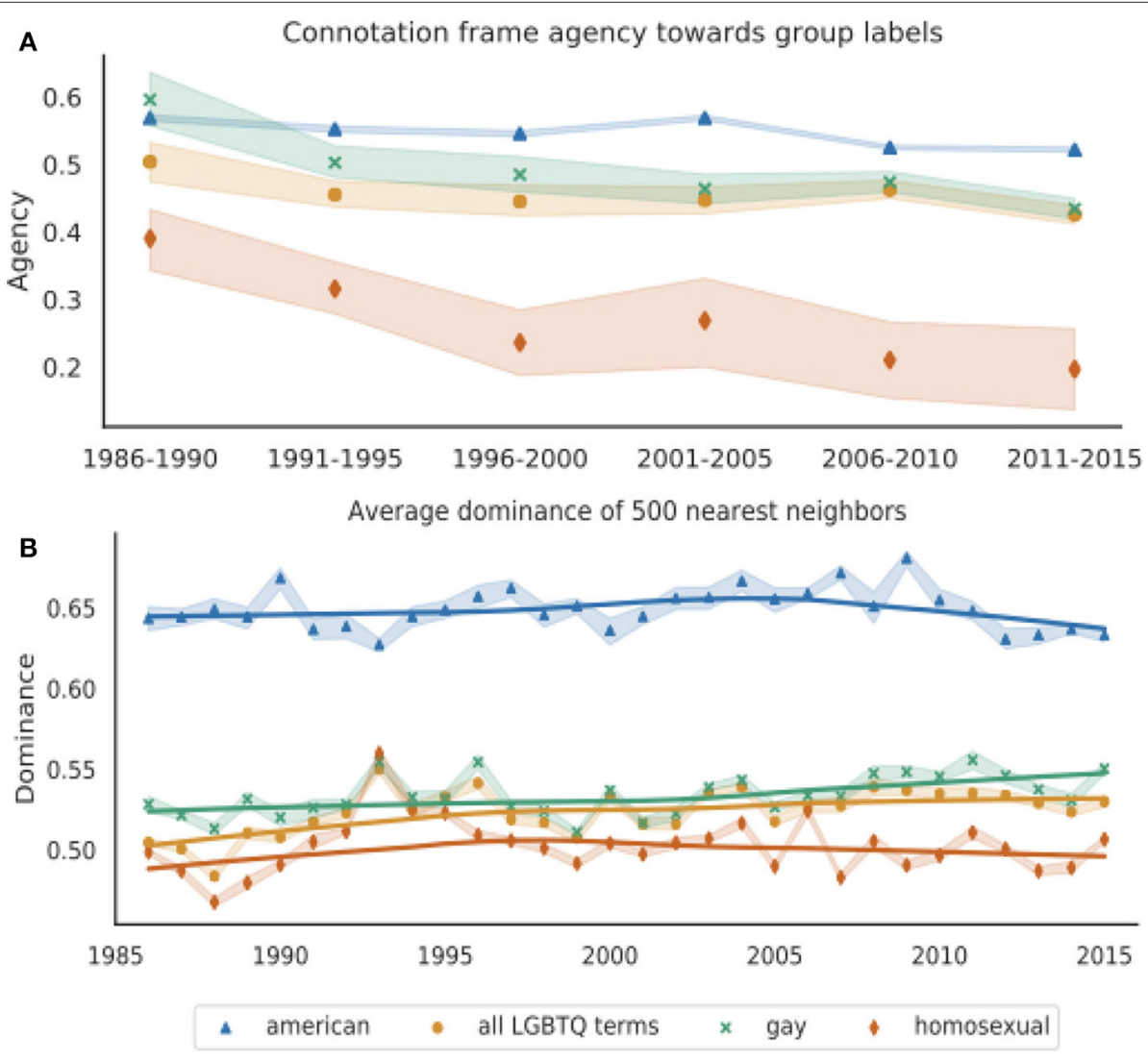

FIGURE 3 | (A) Agency of gay, homosexual, all LGBTQ terms, and American using the Connotation Frames lexicon for agency for all subject-verb-object tuples containing each group label (Sap et al., 2017), calculated over 5-years intervals. An SVO tuple received a score of 1 if the label appears in a positive agency position relative to its head verb and 0 if it does not. (B) Average dominance of 500 nearest words to our representations of gay, homosexual, all LGBTQ terms, and American, averaged over 10 word2vec models trained on New York Times data for each year. Dominance scores for each word come from the word's entry in the NRC VAD Dominance Lexicon (Mohammad, 2018), which range from 0 (least dominance) to 1 (most dominance). For both plots, the shaded bands represent $95 \%$ confidence intervals and the solid lines in (B) are Lowess curves for visualization purposes.

\subsubsection{Word embedding neighbor dominance}

Figure 3B shows the average dominance of each group label's 500 nearest neighbors. American is significantly associated with greater dominance than gay, homosexual, and all LGBTQ terms (Wilcoxon signed-rank test; $p<0.0001$ ), and gay has significantly higher dominance than homosexual $(p<0.0001)$. 
TABLE 8 | Examples where the writer attributes high and low agency toward LGBTQ groups, according to the Connotation Frames lexicon for agency.

\begin{tabular}{|c|c|c|c|}
\hline Agency & Text & SVo & Year \\
\hline High & $\begin{array}{l}\text { Within the close-knit world of professional childbearers, many of whom } \\
\text { share their joys and disillusionments online and in support groups, } \\
\text { gay couples have developed a reputation as especially grateful clients... }\end{array}$ & $\begin{array}{l}\text { S: gay couples } \\
\text { V: developed } \\
\text { O: a reputation }\end{array}$ & 2005 \\
\hline High & $\begin{array}{l}\text { Tonight, the gay rights group Stonewall Democrats will endorse a } \\
\text { candidate for A.G. It's a relatively big prize in the four-man Democratic } \\
\text { primary, given that liberal city voters will have relatively serious sway... }\end{array}$ & $\begin{array}{l}\text { S: the gay rights } \\
\text { group } \\
\text { V: endorse } \\
\text { O: a candidate }\end{array}$ & 2006 \\
\hline Low & $\begin{array}{l}\text { Nigeria's gay men and lesbians regularly face harassment and arrest, } \\
\text { gay activists here say. The criminal code bans acts "against the order } \\
\text { of nature," and imposes sentences of up to } 14 \text { years for those convicted... }\end{array}$ & $\begin{array}{l}\text { S: gay men } \\
\text { V: face } \\
\text { O: harassment }\end{array}$ & 2005 \\
\hline Low & $\begin{array}{l}\text { Much of the debate among military and civilian officials is now focusing } \\
\text { on some version of an approach called "don't ask, don't tell." (...) But } \\
\text { under the "don't tell" element, there would be restrictions on the extent } \\
\text { to which homosexuals could acknowledge their homosexuality. }\end{array}$ & $\begin{array}{l}\text { S: homosexuals } \\
\text { V: acknowledge } \\
\text { O: their } \\
\text { homosexuality }\end{array}$ & 1993 \\
\hline
\end{tabular}

The relevant subject, verb, and object are shown in bold.

While the dominance associated with gay and all LGBTQ terms significantly increased over time $(p<0.0001)$, the dominance associated with homosexual did not significantly change $(p=$ $0.65)$. Furthermore, the average nearest neighbor dominance for homosexual decreased in the most recent 15 years $(p<0.01)$.

Even though dominance may more directly encode power rather than agency, the NRC VAD Dominance Lexicon is useful for operationalizing denial of agency because of the close relationship between these concepts. As with Connotation Frames of agency, these results suggest that LGBTQ groups experience greater denial of agency than the New York Times's in-group American. Both techniques show differences between the labels gay and homosexual, where homosexual is consistently associated with lower agency than gay and further decreases over time. However, these two measurements suggest different temporal dynamics for the denial of agency of LGBTQ people; Connotation Frames' agency slightly decreases for all LGBTQ terms over time, but increases with word embedding neighbor dominance.

\subsubsection{Qualitative Analysis}

\subsubsection{Connotation frames of agency}

We qualitatively investigate the labels assigned by this technique for a sample of paragraphs. In general, the binary labels for positive and negative agency seem reasonably accurate, as shown by the first four example in Table 8. Verbs that attribute high agency to the subject include develop and endorse, suggesting that the LGBTQ-aligned subjects are in control and actively making their own decisions. On the other end, LGBTQ people have low agency when they are the subjects of passive verbs, such as face and acknowledge.

The Connotation Frames lexicon for agency seems to be especially accurate for low agency; we could not find counterexamples in our sample where LGBTQ people were portrayed with high agency but labeled with low agency. However, we found several mischaracterizations where LGBTQ people were labeled as having high agency but are not portrayed as agentive or in control of their own actions.
Our Connotation Frames technique considers the example below to attribute high agency to LGBTQ people because homosexual appears in the subject of the high-agency verb violate; however, homosexual actually modifies relationships, not people themselves. Furthermore, this debate within religion appears to be devoid of input from LGBTQ people and does not portray them as particularly agentive.

- At the same time, it underscored a stark division in Judaism over the place of homosexuals in society. Orthodox rabbinical groups believe that homosexual relationships violate Jewish law...(1996)

\subsubsection{Word embedding neighbor dominance}

Using the VAD Dominance Lexicon to calculate average dominance of each social group label corresponds well to our notion of denial of agency. Because gay's nearest neighbors have a much higher average dominance than homosexual's for most years, we compare words that are nearby neighbors for gay and not homosexual for multiple years' word2vec spaces. Words frequently among the 500 words nearest to gay and not homosexual include high-agency words, such as activist, liberation, advocate, and advocacy, which have dominance scores of $0.877,0.857,0.818$, and 0.731 , respectively. Words frequently among homosexual's 500 nearest neighbors and not gay's include low-agency words, such as submissive (0.173), degrading (0.232), enslavement (0.302), and repressed (0.311).

We additionally investigate the word2vec models corresponding to several outlier years. Homosexual's neighbors have the highest average dominance in 1993, which is likely due to military-related language in debates surrounding the "Don't Ask, Don't Tell" legislation. High-dominance words unique to homosexual's nearest neighbors in 1993 include forces (0.886), military (0.875), enforce (0.836) and troops (0.804). Gay's neighbors' in 1999 have the lowest average dominance than any other year, which is likely connected to Matthew Shepard's death and the subsequent outrage; unique neighbors for gay in 1999 include imprisoned (0.302) and repressed (0.311). 


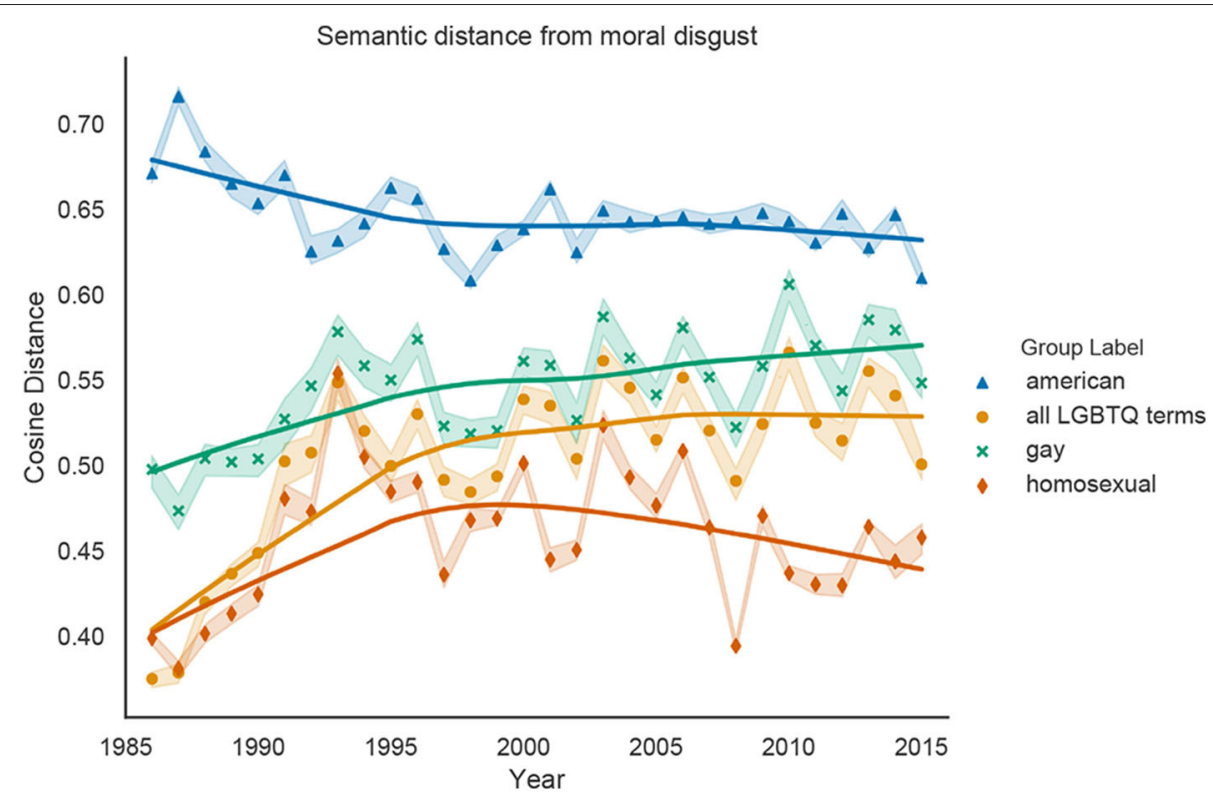

FIGURE 4 | Cosine distance between our representations of gay, homosexual, all LGBTQ terms, and American and the vector representation of the Moral Disgust concept, averaged over 10 word2vec models trained on New York Times data for each year. Increases in cosine distance indicate decreases in Moral Disgust; possible values range from 0 (most closely associated with Moral Disgust) to 1 (least associated with Moral Disgust). Shaded bands represent 95\% confidence intervals and the solid lines are Lowess curves for visualization purposes.

\subsection{Moral Disgust}

\subsubsection{Quantitative Results}

Figure 4 shows the changing relationships between all LGBTQ terms, gay, homosexual and the dehumanizing concept of Moral Disgust. Because the cosine distance between American and Moral Disgust is significantly greater over all years than any LGBTQ representation (Wilcoxon signed-rank test; $p<0.0001$ ), American is the least associated with Moral Disgust. Furthermore, the cosine distance between gay and Moral Disgust is significantly greater than the distance between homosexual and Moral Disgust for every year $(p<0.0001)$, indicating that homosexual is more closely associated with Moral Disgust than gay is. Linear regression analyses show that all LGBTQ terms and gay significantly increase in cosine distance from the Moral Disgust vector $(p<0.0001)$, indicated weakening associations between LGBTQ people and moral disgust over time. On the other hand, the distance between homosexual and Moral Disgust does not change significantly over time $(p=0.54)$, and even decreases after $2000(p<0.05)$.

Overall, these measurements of associations between LGBTQ people and Moral Disgust are consistent with our other operationalizations of dehumanization. All LGBTQ labels are more closely associated with Moral Disgust than the newspaper's in-group term American, but these associations weaken over time, suggesting increased humanization. Notably, the term homosexual has always been more associated with Moral Disgust than the denotationally-similar term gay, and homosexual actually becomes more closely associated with this dehumanizing concept in recent years.

\subsubsection{Qualitative Analysis}

Our analysis of homosexual's changing semantic neighbors from Table 3 has shown that this term has become more associated with immoral concepts, suggesting that moral disgust is a mechanism by which LGBTQ people are dehumanized. Although rarely directly invoked, the connection between LGBTQ people and disgust is supported by the data, such as in the examples shown below, where words belonging to the moral disgust lexicon are in bold. Figure 4 indicates that late 1980s and early 1990s, LGBTQ labels rapidly became more semantically distant from Moral Disgust. This likely reflects decreasing attention to the AIDS epidemic, as many disease-related words are included in the moral disgust lexicon.

- Senator Jesse Helms, the North Carolina Republican who has vigorously fought homosexual rights, wants to reduce the amount of Federal money spent on AIDS sufferers, because, he says, it is their "deliberate, disgusting, revolting conduct" that is responsible for their disease (1995).

- A lawyer named G. Sharp, address unknown, called the cover picture "utterly repulsive." Donald Ingoglia of Sacramento was equally outraged. "Showing two smiling gays on the cover illustrates how sick our society has become," he wrote. "You have my non-lawyer friends falling off their chairs" (1992).

- ...Mr. Robison could be harsh-he yelled in the pulpit and referred to gay men and lesbians as perverts-but Mr. Huckabee was a genial ambassador ... (2008)

- ...When bishops started telling parishioners that their gay and lesbian siblings were sinners, and that family planning was a 


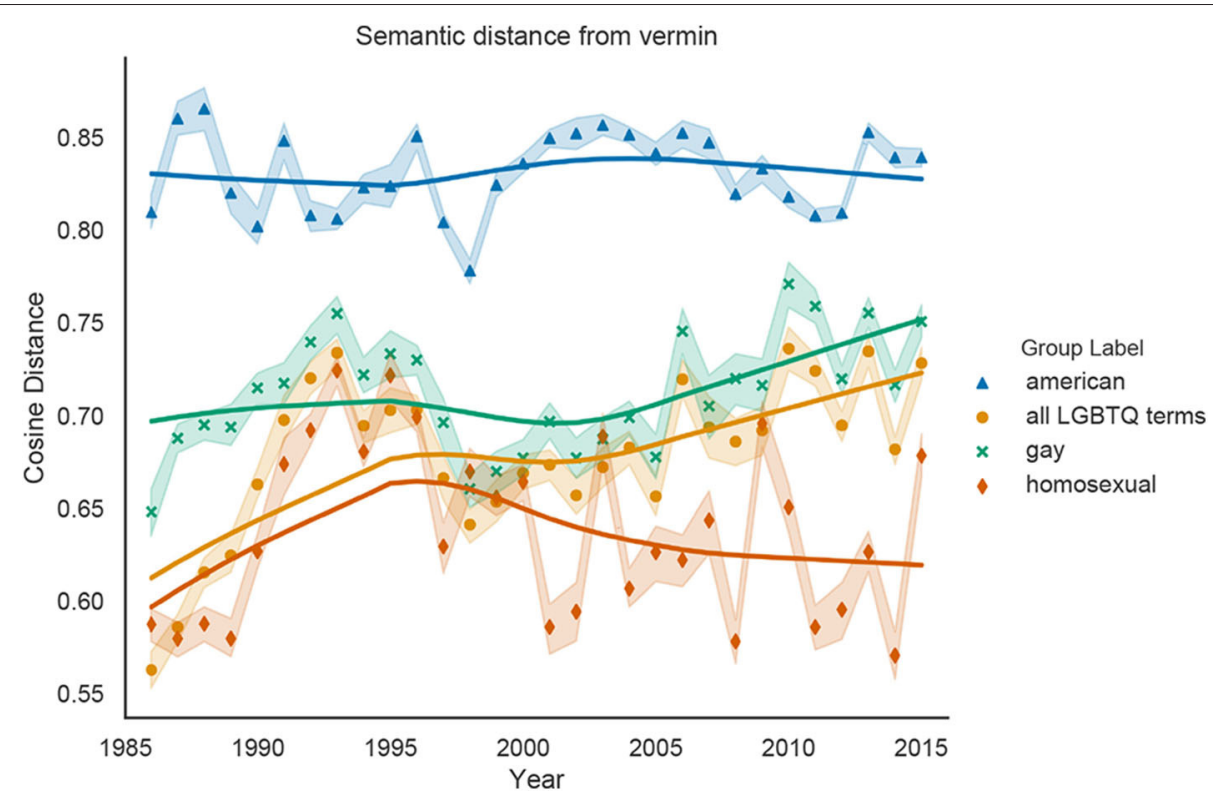

FIGURE 5 | Cosine distance between our representations of gay, homosexual, all LGBTQ terms, and American and the vector representation of the Vermin concept, averaged over 10 word2vec models trained on New York Times data for each year. Possible values for cosine distance range from 0 (most closely associated with Vermin) to 1 (least associated with Vermin). Shaded bands represent 95\% confidence intervals, and the solid lines are Lowess curves for visualization purposes.

grievous wrong, people stopped listening to them-for good reason (2013).

\subsection{Vermin as a Dehumanizing Metaphor}

\subsubsection{Quantitative Results}

Figure 5 shows the relationships between LGBTQ labels (and American) and the dehumanizing vermin metaphor, quantified as the cosine distance between the labels' word2vec vectors and a Vermin concept representation, which is the centroid of multiple vermin-related words. As with Moral Disgust, the in-group term American is further away from Vermin over all years than any LGBTQ term (Wilcoxon signed-rank test; $p<0.0001$ ). The cosine distance between gay and Vermin is also greater than between homosexual and Vermin $(p<0.0001)$, indicating that homosexual is more closely associated with the dehumanizing vermin metaphor than gay is. Furthermore, while all LGBTQ terms and gay become more semantically distant from Vermin over time, $(p<0.0001)$, the association between Vermin and homosexual does not significantly change over time $(p=0.13)$.

This measure of the implicit vermin metaphor reveals similar patterns as the other dehumanization measures. Overall, LGBTQ groups are more associated with vermin than the comparison group American, but this association weakens over time, suggesting increased humanization. In addition, homosexual has become a more dehumanizing term, with stronger associations with vermin than other LGBTQ labels.

\subsubsection{Qualitative Analysis}

Metaphors comparing humans to vermin have been especially prominent in dehumanizing groups throughout history (Haslam,
2006; Steuter and Wills, 2010). Although no New York Times writers directly compare LGBTQ people to vermin, this metaphor may be invoked in more subtle ways. There are only three paragraphs in the LGBTQ corpus that explicitly mention vermin in order to criticize the LGBTQ people-as-vermin metaphor. Nevertheless, these paragraphs point to the existence of this metaphor.

- Since gay women can't be stigmatized en masse with AIDS, the council had to use real ingenuity to prove that they, too, are vermin at "much greater risk from one another" than from gay-bashers ... (1998)

- "The equating of gay men to vermin is appalling," Addessa said from Philadelphia. "We need to encourage the Eagles and Owens to make a public apology and for the Eagles to publicly discipline Owens. These comments that equate gay men to some inferior life form do real harm, creating a cultural environment which justifies violence against gay and lesbian people (2004).

- In $3 \mathrm{~h}$ at training camp Tuesday, he hustled vigorously through practice, eagerly signed autographs for visiting military personnel and tried to explain incendiary remarks that appeared in a magazine regarding the sexual orientation of a former teammate in San Francisco, words that seemed to compare gays to rodents (2004).

\section{HUMAN EVALUATION OF VECTOR-BASED MEASURES}

Our vector-based methods can directly capture associations between LGBTQ people and dehumanizing concepts. However, 
findings from these methods are difficult to interpret, as discussed in earlier qualitative analysis sections. Furthermore, while the NRC VAD Lexicon and the Connotation Frames Lexicons have been evaluated in prior work (Rashkin et al., 2016; Sap et al., 2017; Mohammad, 2018), our vector-based methods have not. Thus, we recruit humans from Amazon Mechanical Turk (MTurk) to quantitatively evaluate our four vector-based measures: word embedding neighbor valence (for negative evaluation of a target group), word embedding neighbor dominance (for denial of agency), semantic distance from the concept of moral disgust, and semantic distance from the concept of vermin.

Although these four measures rely on vector representations of LGBTQ labels and not individual paragraphs, we use paragraphs as the unit of analysis for our evaluation in order for the task to be feasible for human annotators. In section 6.1, we describe how we use our vector-based methods to obtain the most and least dehumanizing paragraphs for each dehumanization component. We discuss the MTurk task design in section 6.2 and results in section 6.3 .

\subsection{Identifying the Most (De)humanizing Paragraphs \\ 6.1.1. Word Embedding Neighbor Valence and Dominance}

Our word embedding neighbor valence and dominance methods are proxies for measuring the negative evaluation of the target group and denial of agency dimensions of dehumanization, respectively. They directly estimate the valence and dominance scores for LGBTQ terms based on NRC VAD entries for each term's semantic neighbors.

To obtain full paragraphs corresponding to the most and least dehumanizing extremes of negative evaluation of a target group, we first train word2vec on the entire New York Times dataset using the same hyperparameters as in section 3.1.3. Let $N$ be the nearest 500 words to the representation of all LGBTQ terms in this vector space, and let $V$ and $D$ be the full NRC Valence and Dominance Lexicons. We define subset lexicons, $V_{s}=N \cap V$ and $D_{s}=N \cap D ; V_{s}$ and $D_{s}$ are the subsets of the NRC Valence and Dominance Lexicons containing only words that neighbor all LGBTQ terms. We calculate neighbor valence scores for each paragraph $P$ as $\frac{1}{|P|} \Sigma_{w \in P} V_{s}[w]$, where $|P|$ is the total number of tokens in $P$ and $V_{s}[w]$ is the valence score of $w$. Similarly, we calculate neighbor dominance scores as $\frac{1}{|P|} \Sigma_{w \in P} D_{s}[w]$.

For human evaluation, we consider paragraphs with the highest and lowest scores for neighbor valence and neighbor dominance. We remove paragraphs containing fewer than 15 or more than 75 words. Because our case study focuses on the words $g a y(s)$ and homosexual(s), we further restrict our sample to paragraphs containing these terms.

\subsubsection{Moral Disgust and Vermin Metaphor}

We measure implicit associations of LGBTQ groups with moral disgust and vermin by calculating the cosine distance between LGBTQ terms' vectors and vector representations of moral disgust and vermin. Thus, we identify paragraphs corresponding to the most and least dehumanizing extremes by comparing the cosine distance between paragraph embeddings and the Moral Disgust and Vermin concept vectors. We create each paragraph's embedding by calculating the tfidf-weighted average of all words' vectors and removing the first principal component, which improves the quality of sentence and document embeddings (Arora et al., 2019).

We select the paragraphs that are the closest (most semantically similar) and furthest from the Moral Disgust and Vermin vectors based on cosine distance. As in section 6.1.1, we limit our sample to paragraphs containing between 15 and 75 words and either the term gay(s) or homosexual(s).

\subsection{MTurk Task Design}

As discussed in our qualitative analyses, journalistic text captures numerous perspectives, not only from journalists themselves, but also from people quoted and people or groups described within the text. While our current computational methods do not disambiguate these perspectives, human evaluation can provide insights into whose perspectives primarily drive our findings about dehumanization. Thus, we manually divide each measure's most and least dehumanizing paragraphs into three categories based on whose views are most prominent: the author, a person quoted or paraphrased, or a person/group mentioned or described within the text. For each measure, our final sample for human evaluation consists of the 20 most humanizing and 20 most dehumanizing paragraphs within each of the three "viewpoint" categories, yielding 120 paragraphs for each vectorbased measure.

MTurk workers read a paragraph and answered a question about the attitudes of the author, person quoted, or people mentioned/described in the text. Table 9 shows four examples, the dehumanization component that they correspond to, whether they are ranked high (most dehumanizing) or low (least dehumanizing), the most prominent viewpoint, and the exact question that workers answered. The question depends on which dehumanization component's measure is being evaluated. In addition, we include the actual name of people quoted or mentioned in order to simplify the task. Each question is answered with a 5-point Likert scale with endpoints specified in the task. For the negative evaluation and denial of agency questions, 1 is the most dehumanizing option and 5 is the most humanizing option, but the opposite is the case for vermin and moral disgust. As a post-processing step, we reverse the scale for the latter so higher values always correspond to more humanizing views.

Three MTurk workers completed each task. Workers were located in the United States, already completed at least 1,000 MTurk tasks, and have an approval rate of at least $98 \%$. Each task took $\sim 20-25 \mathrm{~s}$ and workers were compensated $\$ 0.05$. To avoid confusion with multiple question formulations, we published the tasks for each dehumanization component separately.

\subsection{Human Evaluation Results}

The results from the MTurk study, shown in Figure 6, largely support our use of vector-based measures. Paragraphs with the highest neighbor valence were judged to hold more positive evaluations of gay people $(p<0.0001)$. Paragraphs whose 
TABLE 9 | Examples of four paragraphs annotated by MTurk workers, one for each dehumanization component.

\begin{tabular}{|c|c|c|c|c|}
\hline Paragraph & Component & Extreme & Viewpoint & Question \\
\hline $\begin{array}{l}\text { Some people think that equality can be achieved by } \\
\text { offering gays civil unions in lieu of marriage. Civil } \\
\text { unions are not a substitute for marriage. Separate } \\
\text { rights are never equal rights. }\end{array}$ & $\begin{array}{l}\text { Negative } \\
\text { evaluation }\end{array}$ & Low & Author & $\begin{array}{l}\text { How does the author feel } \\
\text { about gay people? }\end{array}$ \\
\hline $\begin{array}{l}\text { "I also learned it was possible to be black and gay," } \\
\text { Mr. Freeman said. "The first black gay I met, I } \\
\text { didn't believe it. I thought you could only be a } \\
\text { member of one oppressed minority." }\end{array}$ & $\begin{array}{l}\text { Denial of } \\
\text { agency }\end{array}$ & High & $\begin{array}{l}\text { Person } \\
\text { quoted }\end{array}$ & $\begin{array}{l}\text { To what extent does Mr. Freeman } \\
\text { think that gay people are able to } \\
\text { control their own actions and decisions? }\end{array}$ \\
\hline $\begin{array}{l}\text { In a speech exceptional for its deep emotion and } \\
\text { sharp message, Ms. Fisher implicitly rebuked those } \\
\text { in her party who have regarded the sickness as a } \\
\text { self-inflicted plague earned by immoral behavior- } \\
\text { homosexual sex or intravenous drug abuse. }\end{array}$ & $\begin{array}{l}\text { Moral } \\
\text { disgust }\end{array}$ & High & $\begin{array}{l}\text { Person } \\
\text { mentioned }\end{array}$ & $\begin{array}{l}\text { To what extent does Ms. Fisher's } \\
\text { party consider gay people to be } \\
\text { disgusting or repulsive? }\end{array}$ \\
\hline $\begin{array}{l}\text { The Supreme Court on Tuesday was deeply divided } \\
\text { over one of the great civil rights issues of the age, } \\
\text { same-sex marriage. But Justice Anthony M. Kennedy, } \\
\text { whose vote is probably crucial, gave gay rights } \\
\text { advocates reasons for optimism based on the tone } \\
\text { and substance of his questions. }\end{array}$ & Vermin & Low & $\begin{array}{l}\text { Person } \\
\text { mentioned }\end{array}$ & $\begin{array}{l}\text { Vermin are animals that carry disease or } \\
\text { cause other problems for humans. } \\
\text { Examples include rats and cockroaches. } \\
\text { To what extent does [the author] consider } \\
\text { gay people to be vermin-like? }\end{array}$ \\
\hline
\end{tabular}

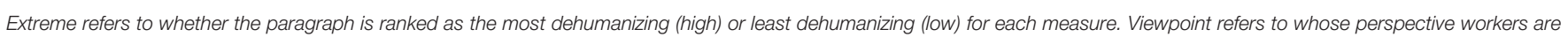
asked to reason about. The question that MTurk workers answer is modified based on both the dehumanization component and the viewpoint.

embeddings are nearest to the Moral Disgust concept vector are judged to express stronger views of gay people as "disgusting" or "repulsive" compared to the furthest paragraphs $(p<0.0001)$. Similarly, paragraphs nearest to Vermin concept consider gay people to be more vermin-like than the paragraphs furthest away $(p<0.0001)^{9}$.

The only component that does not follow these expected results is denial of agency, where paragraphs with highest and lowest neighbor dominance are not judged to be significantly different $(p=0.19$ ). This may reflect that using a lexicon for dominance is not a perfect proxy for the more nuanced concept of agency. Another possible explanation is the inherent complexity in measuring denial of agency. While the other components are already challenging by requiring an annotator to reason about another person's attitudes toward the target group, assessing denial of agency is even more complicated, as it requires an annotator to reason about another person's perceptions of the cognitive capabilities of members of the target group.

The bottom row of Figure 6 separates the results based on whose viewpoint MTurk workers are asked to reason about: the paragraph's author, the people quoted, or the people mentioned in the text. This reveals a strikingly consistent pattern; the difference between the two extremes is largest when workers are asked about the people mentioned, smallest when asked about the author, and in-between when asked about people quoted. This suggests that dehumanizing representations of LGBTQ people in the New York Times may be most driven by descriptions

\footnotetext{
${ }^{9}$ We evaluate our methods in this way instead of using traditional precision and recall metrics because annotators rated each example on a 5-point scale, so binarizing annotations risks losing valuable information. Precision, recall, and F1 scores for each component can be found in the Supplementary Material.
}

about other people's attitudes, and to a lesser extent, direct quotes and paraphrases.

\section{DISCUSSION}

Our framework for the computational linguistic analysis of dehumanization involves identifying major dimensions of dehumanization from social psychology literature, proposing linguistic correlates for each dimension, and developing robust and interpretable computational methods to quantify these correlates. We apply this framework to study the dehumanization of LGBTQ people in the New York Times from 1986 to 2015. We measure four dimensions of dehumanization: negative evaluations of a target group, denial of agency, moral disgust, and (implicit) invocations of vermin metaphors. Overall, we find increasingly humanizing descriptions of LGBTQ people over time. LGBTQ people have become more associated with positive emotional language, suggesting that negative evaluations of the target group have diminished. LGBTQ people have become more associated with higherdominance words, suggesting decreasing denial of agency, although this finding was not replicated with the verb-centric "Connotation Frames" measurement. Furthermore, labels for LGBTQ people have moved further away from the concepts of moral disgust and vermin within distributional semantic representations, suggesting that harmful associations between LGBTQ people and these dehumanizing concepts have weakened over time.

Despite these trends, the labels gay and homosexual exhibit strikingly different patterns. Homosexual is associated with more negative language than gay, suggesting more negative evaluations of people described as homosexual than gay. 


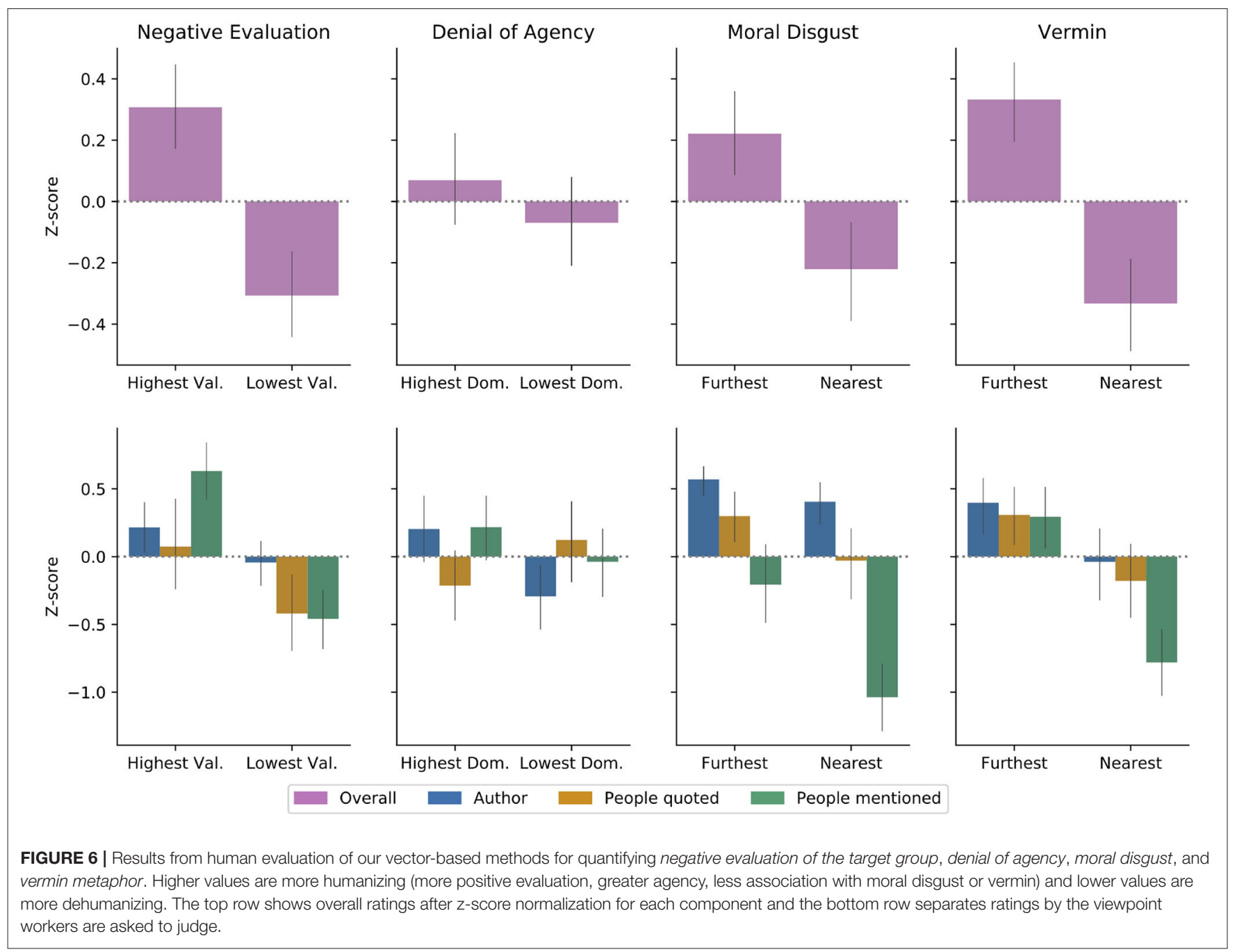

Homosexual is also associated with greater denial of agency, and has stronger connections to moral disgust and vermin than gay. Unlike for other LGBTQ labels, discussions of homosexual people have not become more humanizing over time, and several techniques even suggest that homosexual has become used in more dehumanizing contexts since 2000. Through its repeated use in these contexts, the use of the word homosexual appears to have emerged as an index of more dehumanizing attitudes toward LGBTQ people than other labels. Despite the denotational similarity between homosexual and gay, our computational techniques tracks the stark divergence in social meanings.

We restrict our analysis to the lexical level for ease of interpretability, and leveraged a diverse array of existing resources, including the NRC VAD lexicon (Mohammad, 2018), Connotation Frames lexicons (Rashkin et al., 2016; Sap et al., 2017), and the Moral Foundations Dictionary (Graham et al., 2009). For negative evaluations of a target group and denial of agency, we propose multiple different techniques that vary in accuracy and interpretability. Word-counting methods are often inaccurate due to their simplicity but their results are easily interpretable, while embedding-based methods suffer the opposite problem. Carefully considering the tradeoff between model quality and interpretability is especially important in work that seeks to characterize complex and sensitive social phenomena, such as dehumanization.

\subsection{Limitations and Future Work}

As the first attempt to computationally analyze dehumanization, this work has many limitations. While we demonstrate how the proposed techniques capture linguistic signals of dehumanization, our qualitative and quantitative evaluation suggest that the findings may be driven more by events and attitudes of people described in the text rather than the journalists' own views. An exciting area of future work could involve developing more sophisticated methods to disambiguate the writer's attitudes, attitudes of people mentioned or quoted, and events, while recognizing that each of these could contribute 
to the overall representation of marginalized groups in the media. In addition, the present work uses word2vec since all known affective lexicons are type-level, but contextualized embedding-based methods have great potential for more nuanced analyses of dehumanizing language by leveraging tokenlevel representations (Devlin et al., 2018; Peters et al., 2018).

Our framework could be expanded to include more insights from dehumanization theory. Beyond the four components discussed in this article, social psychology research has identified other cognitive processes that contribute to dehumanization, including psychological distancing, essentialism (the perception that the target group has some essence that makes them categorically and fundamentally different), and denial of subjectivity (neglect of a target group member's personal feelings and experiences) (Rothbart and Taylor, 1992; Nussbaum, 1999; Graf et al., 2013; Haslam and Stratemeyer, 2016). Scholars also differentiate between two forms of dehumanization, animalistic (likening humans to animals) and mechanistic (likening humans to inanimate objects or machines), which may differ substantially in their linguistic expressions (Haslam, 2006).

For simplicity and ease of interpretation, we quantify lexical cues of dehumanization. However, our understanding of dehumanizing language would be enriched by considering linguistic features beyond the lexicon. For example, Acton (2014) has shown that definite plurals in English (e.g., the gays) have a unique social and pragmatic effect compared to bare plurals (e.g., gays) by packaging individual entities into one monolith and setting this group apart from the speaker. Indexing a speaker's non-membership in the group being discussed creates social distance between the speaker and group (Acton, 2014), which makes it likely that using definite plurals to label marginalized social groups plays an important role in dehumanization. Similarly, examining non-lexical signals could help us capture elements of dehumanization not easily identifiable with lexical resources alone. For example, a group label's word class (e.g., gay as a noun or adjective) may have implications for essentialism, as adjectives simply name attributes to some entity, while nouns explicitly state the entity's category membership and encapsulates other stereotypes associated with that category (Wierzbicka, 1986; Hall and Moore, 1997; Graf et al., 2013; Palmer et al., 2017). We furthermore believe that incorporating discourse-level analysis, such as examining the role of direct quotes in an article and who is being quoted, could provide informative insights that could address some limitations discussed earlier.

We support our proposed framework with a case study of LGBTQ representation in the New York Times. This case study is limited as an analysis of the dehumanization of LGBTQ people in the media. We only investigate one data source, which does not capture the entirety of media discourse about LGBTQ people. Furthermore, we only study newspaper articles written in (Standard) American English. Future work could focus on crosslinguistic comparisons of dehumanizing language and assess how well our measures generalize to other languages. Finally, the case study focuses on the labels gay and homosexual due to data availability. As a consequence, we have less understanding about the differences and changes in representations of LGBTQ people who do not identify with these labels.

The primary aim of this paper is to develop a computational framework for analyzing dehumanizing language toward targeted groups. While our in-depth case study focuses on one particular social group, this framework can be generalized to study dehumanization across a wide variety of social groups, and this could be a fruitful area of future work. For example, Asians have faced increased prejudice and dehumanization since the beginning of the COVID-19 pandemic (Van Bavel et al., 2020; Vidgen et al., 2020; Ziems et al., 2020). Our framework could be applied to understand who dehumanizes these populations in both news and social media, and how the degree of dehumanization changes over time or varies by region. This framework could provide a nuanced view into the shifting nature of dehumanization toward Asians. For example, the "Asians are good at math" stereotype may have led dehumanization via denial of agency or denial of subjectivity (Shah, 2019). However, stereotypes of Asians as COVID-19 carriers may have made moral disgust and associations with vermin more salient mechanisms of dehumanization. In our case study, we use computational measures of dehumanizing language to show how the terms gay and homosexual have diverged in meaning. This method of demonstrating how denotationally similar items differ in connotation can also generalize to other issues and social groups. For example, we may expect labeling COVID-19 as the Wuhan Virus or Chinese Virus may be associated with greater dehumanization of Asians than the names COVID-19 or SARS-CoV-2 (Van Bavel et al., 2020; Xu and Liu, 2020).

\subsection{Ethical Implications}

We hope to draw attention to issues of media representation of marginalized groups and to eventually help make the online world a safer and kinder place. An important part of this mission is to acknowledge the ethical implications and potential issues of our own work.

The methods that we use to quantify dehumanization are themselves biased and potentially harmful. For example, we show in section 5.2.1.1 that the lexicon used to measure valence contains its own anti-LGBTQ biases by considering LGBTQ group labels to be primarily negative/unpleasant. We also train word2vec models on New York Times data, which presents biases. Though models trained on biased data are typically concerning due to harmful downstream effects (Bolukbasi et al., 2016), we leverage this data as a resource for uncovering human biases and understanding how biases emerge in the media.

Another concern of this work in our computational methods to represent human beings. Representing people as sequences of numbers (especially in our vector-based experiments) is inherently dehumanizing. While we hope that this work will humanize and empower marginalized groups, we acknowledge that it can also have effect of perpetuating their dehumanization.

Other ethical implications of this project appear within our case study. We do not include LGBTQ labels, such as queer or trans, which often had different meanings and were found in unrelated contexts in earlier years. Furthermore, our 
analysis uses an aggregated representation for LGBTQ people, which unintentionally minimizes the vast diversity of social identities encompassed within this umbrella. We highlight striking temporal changes and differences between gay and homosexual, which were chosen because these labels were wellrepresented in all years. However, emphasizing these labels at the expense of others may contribute to the erasure of people who are marginalized even within LGBTQ communities.

\section{CONCLUSION}

This work is the first known computational linguistic study of dehumanization, and provides contributions to multiple fields. The proposed framework and techniques to quantify salient components of dehumanization can shed light on linguistic variation and change in discourses surrounding marginalized groups. Furthermore, these tools for large-scale analysis have potential to complement smaller-scale psychological studies of dehumanization. Finally, this work has implications for automatically detecting and understanding media bias and abusive language online.

\section{DATA AVAILABILITY STATEMENT}

The datasets generated for this study are available on request to the corresponding author.

\section{REFERENCES}

Acton, E. K. (2014). Pragmatics and the social meaning of determiners (Ph.D. thesis), Stanford University, Stanford, CA, United States.

Alorainy, W., Burnap, P., Liu, H., and Williams, M. L. (2019). "The enemy among us" detecting cyber hate speech with threats-based othering language embeddings. ACM Trans. Web 13, 1-26. doi: 10.1145/3324997

Arora, S., Liang, Y., and Ma, T. (2019). A simple but tough-to-beat baseline for sentence embeddings. In 5th International Conference on Learning Representations, ICLR 2017. (Toulon).

Barnhurst, K. G., and Mutz, D. (1997). American journalism and the decline in event-centered reporting. J. Commun. 47, 27-53. doi: 10.1111/j.1460-2466.1997.tb02724.x

Bar-Tal, D. (1990). Causes and consequences of delegitimization: models of conflict and ethnocentrism. J. Soc. Issues 46, 65-81. doi: 10.1111/j.1540-4560.1990.tb00272.x

Baumer, E., Elovic, E., Qin, Y., Polletta, F., and Gay, G. (2015). "Testing and comparing computational approaches for identifying the language of framing in political news," in Proceedings of the 2015 Conference of the North American Chapter of the Association for Computational Linguistics: Human Language Technologies (Denver, CO), 1472-1482. doi: 10.3115/v1/N15-1171

Blodgett, S. L., and O'Connor, B. (2017). Racial disparity in natural language processing: a case study of social media african-american english. arXiv[Preprint].arXiv:1707.00061

Bolukbasi, T., Chang, K.-W., Zou, J. Y., Saligrama, V., and Kalai, A. T. (2016). "Man is to computer programmer as woman is to homemaker? Debiasing word embeddings," In Proceedings of the 30th International Conference on Neural Information Processing Systems (Barcelona), 4356-64.

Boydstun, A. E., Card, D., Gross, J., Resnick, P., and Smith, N. A. (2014). Tracking the Development of Media Frames Within and Across Policy Issues.

Boydstun, A. E., Gross, J. H., Resnik, P., and Smith, N. A. (2013). "Identifying media frames and frame dynamics within and across policy issues," in New Directions in Analyzing Text as Data Workshop (London).

\section{AUTHOR CONTRIBUTIONS}

JM, YT, and DJ collaborated on the conception and design of the study, read, and revised the manuscript. JM prepared the data, conducted the case study analysis, and conducted statistical analysis. A first draft of the paper was written by JM. All authors approved the submitted version.

\section{ACKNOWLEDGMENTS}

We would like to thank Rob Voigt, Chris Potts, Penny Eckert, David Jurgens, Ceren Budak, Anna-Marie Sprenger, and Ismael Mendoza for their insightful contributions and feedback. We would also like to thank our audience at NWAV-46, who provided helpful feedback on an earlier version of this project. Finally, we thank our reviewers for their thoughtful and valuable comments. This research was supported in part by Public Interest Technology University Network Grant No. NVF-PITU-Carnegie Mellon University-Subgrant-009246-2019-10-01.

\section{SUPPLEMENTARY MATERIAL}

The Supplementary Material for this article can be found online at: https://www.frontiersin.org/articles/10.3389/frai.2020. 00055/full\#supplementary-material

Breitfeller, L., Ahn, E., Jurgens, D., and Tsvetkov, Y. (2019). "Finding microaggressions in the wild: a case for locating elusive phenomena in social media posts," in Proceedings of the 2019 Conference on Empirical Methods in Natural Language Processing and the 9th International Joint Conference on Natural Language Processing (EMNLP-IJCNLP) (Hong Kong: Association for Computational Linguistics), 1664-1674. doi: 10.18653/v1/ D19-1176

Buckels, E. E., and Trapnell, P. D. (2013). Disgust facilitates outgroup dehumanization. Group Process. Intergr. Relat. 16, 771-780. doi: $10.1177 / 1368430212471738$

Burnap, P., and Williams, M. L. (2016). Us and them: identifying cyber hate on twitter across multiple protected characteristics. EPJ Data Sci. 5:11. doi: 10.1140/epjds/s13688-016-0072-6

Caliskan, A., Bryson, J. J., and Narayanan, A. (2017). Semantics derived automatically from language corpora contain human-like biases. Science 356, 183-186. doi: 10.1126/science.aal4230

Card, D., Boydstun, A., Gross, J. H., Resnik, P., and Smith, N. A. (2015). "The media frames corpus: annotations of frames across issues," in Proceedings of the 53rd Annual Meeting of the Association for Computational Linguistics and the 7th International Joint Conference on Natural Language Processing (Volume 2: Short Papers) (Beijing), 438-444. doi: 10.3115/v1/P15-2072

Demszky, D., Garg, N., Voigt, R., Zou, J., Shapiro, J., Gentzkow, M., et al. (2019). "Analyzing polarization in social media: method and application to tweets on 21 mass shootings," in Proceedings of the 2019 Conference of the North American Chapter of the Association for Computational Linguistics: Human Language Technologies, Volume 1 (Long and Short Papers) (Minneapolis, MN), 2970-3005. doi: 10.18653/v1/N19-1304

Devlin, J., Chang, M.-W., Lee, K., and Toutanova, K. (2018). Bert: Pretraining of deep bidirectional transformers for language understanding. arXiv[Preprint].arXiv: 1810.04805.

Dinakar, K., Jones, B., Havasi, C., Lieberman, H., and Picard, R. (2012). Common sense reasoning for detection, prevention, and mitigation of cyberbullying. ACM Trans. Interact. Intell. Syst. 2:30. doi: 10.1145/2362394.2362400 
Dinu, G., Lazaridou, A., and Baroni, M. (2014). Improving zero-shot learning by mitigating the hubness problem. arXiv[Preprint].arXiv: 1412.6568.

ElSherief, M., Kulkarni, V., Nguyen, D., Wang, W. Y., and Belding, E. (2018). "Hate lingo: a target-based linguistic analysis of hate speech in social media," in Twelfth International AAAI Conference on Web and Social Media (Palo Alto, CA).

Entman, R. M. (1993). Framing: toward clarification of a fractured paradigm. J. Commun. 43, 51-58. doi: 10.1111/j.1460-2466.1993.tb01304.x

Esses, V. M., Medianu, S., and Lawson, A. S. (2013). Uncertainty, threat, and the role of the media in promoting the dehumanization of immigrants and refugees. J. Soc. Issues 69, 518-536. doi: 10.1111/josi.12027

Fast, E., and Horvitz, E. (2016). Long-term trends in the public perception of artificial intelligence. arXiv[Preprint].arXiv:1609.04904.

Field, A., Bhat, G., and Tsvetkov, Y. (2019). "Contextual affective analysis: a case study of people portrayals in online\# metoo stories," in Proceedings of the International AAAI Conference on Web and Social Media, Vol. 13 (Munich), 158-169.

Field, A., Kliger, D., Wintner, S., Pan, J., Jurafsky, D., and Tsvetkov, Y. (2018). "Framing and agenda-setting in russian news: a computational analysis of intricate political strategies," in Proceedings of the 2018 Conference on Empirical Methods in Natural Language Processing (Brussels), 3570-3580. doi: 10.18653/v1/D18-1393

Field, A., and Tsvetkov, Y. (2019). "Entity-centric contextual affective analysis," in 57th Annual Meeting of the Association for Computational Linguistics (ACL 2019) (Florence). doi: 10.18653/v1/P19-1243

Gallup (2019). Gay and Lesbian Rights. Available online at: http://news.gallup.com/poll/1651/gay-lesbian-rights.aspx

Garg, N., Schiebinger, L., Jurafsky, D., and Zou, J. (2018). Word embeddings quantify 100 years of gender and ethnic stereotypes. Proc. Natl. Acad. Sci. U.S.A. 115, E3635-E3644. doi: 10.1073/pnas.1720347115

Garten, J., Boghrati, R., Hoover, J., Johnson, K. M., and Dehghani, M. (2016). "Morality between the lines: detecting moral sentiment in text," in Proceedings of IJCAI 2016 Workshop on Computational Modeling of Attitudes (New York, NY). Retrieved from: http://mortezadehghani.net/wpcontent/uploads/morality-lines-detecting.pdf

Gentzkow, M., and Shapiro, J. M. (2010). What drives media slant? Evidence from US daily newspapers. Econometrica 78, 35-71. doi: 10.3982/ECTA7195

Goff, P. A., Eberhardt, J. L., Williams, M. J., and Jackson, M. C. (2008). Not yet human: implicit knowledge, historical dehumanization, and contemporary consequences. J. Pers. Soc. Psychol. 94:292. doi: 10.1037/0022-3514.94.2.292

Graf, S., Bilewicz, M., Finell, E., and Geschke, D. (2013). Nouns cut slices: effects of linguistic forms on intergroup bias. J. Lang. Soc. Psychol. 32, 62-83. doi: 10.1177/0261927X12463209

Graham, J., Haidt, J., and Nosek, B. A. (2009). Liberals and conservatives rely on different sets of moral foundations. J. Pers. Soc. Psychol. 96:1029. doi: 10.1037/a0015141

Greene, S., and Resnik, P. (2009). "More than words: syntactic packaging and implicit sentiment," in Proceedings of Human Language Technologies: The 2009 Annual Conference of the North American Chapter of the Association for Computational Linguistics (Boulder, CO: Association for Computational Linguistics), 503-511. doi: 10.3115/1620754.1620827

Haidt, J., and Graham, J. (2007). When morality opposes justice: conservatives have moral intuitions that liberals may not recognize. Soc. Justice Res. 20, 98-116. doi: 10.1007/s11211-007-0034-z

Hall, D. G., and Moore, C. E. (1997). Red bluebirds and black greenflies: preschoolers' understanding of the semantics of adjectives and count nouns. J. Exp. Child Psychol. 67, 236-267. doi: 10.1006/jecp.1997.2404

Hamilton, W. L., Leskovec, J., and Jurafsky, D. (2016). Diachronic word embeddings reveal statistical laws of semantic change. arXiv[Preprint].arXiv:1605.09096. doi: 10.18653/v1/P16-1141

Harris, L. T., and Fiske, S. T. (2015). Dehumanized perception. Z. Psychol. 219, 175-181. doi: 10.1027/2151-2604/a000065

Haslam, N. (2006). Dehumanization: an integrative review. Pers. Soc. Psychol. Rev. 10, 252-264. doi: 10.1207/s15327957pspr1003_4

Haslam, N., and Stratemeyer, M. (2016). Recent research on dehumanization. Curr. Opin. Psychol. 11, 25-29. doi: 10.1016/j.copsyc.2016.03.009

Hinds, J. (1977). Paragraph structure and pronominalization. Paper Linguist. 10, 77-99. doi: 10.1080/08351819709370440
Hodson, G., and Costello, K. (2007). Interpersonal disgust, ideological orientations, and dehumanization as predictors of intergroup attitudes. Psychol. Sci. 18, 691-698. doi: 10.1111/j.1467-9280.2007.01962.x

Katajamaki, H., and Koskela, M. (2006). "The rhetorical structure of editorials in english, swedish and finnish business newspapers," in Teoksessa Proceedings of the 5th International Aelfe Conference (Zaragoza), 215-19.

Kiritchenko, S., and Mohammad, S. (2018). "Examining gender and race bias in two hundred sentiment analysis systems," in Proceedings of the Seventh Joint Conference on Lexical and Computational Semantics (New Orleans, LA), 43-53. doi: 10.18653/v1/S18-2005

Kteily, N., Bruneau, E., Waytz, A., and Cotterill, S. (2015). The ascent of man: theoretical and empirical evidence for blatant dehumanization. J. Pers. Soc. Psychol. 109:901. doi: 10.1037/pspp0000048

Kulkarni, V., Al-Rfou, R., Perozzi, B., and Skiena, S. (2015). "Statistically significant detection of linguistic change," in Proceedings of the 24th International Conference on World Wide Web (Florence: International World Wide Web Conferences Steering Committee), 625-635. doi: 10.1145/2736277. 2741627

Levy, O., and Goldberg, Y. (2014). "Dependency-based word embeddings," in Proceedings of the 52nd Annual Meeting of the Association for Computational Linguistics (Volume 2: Short Papers) (Baltimore, MD), 302-308. doi: 10.3115/v1/P14-2050

Manzini, T., Lim, Y. C., Tsvetkov, Y., and Black, A. W. (2019). Black is to criminal as caucasian is to police: detecting and removing multiclass bias in word embeddings. arXiv[Preprint].arXiv: 1904.04047. doi: 10.18653/v1/N19-1062

Marshall, S. R., and Shapiro, J. R. (2018). When "scurry" vs."hurry" makes the difference: vermin metaphors, disgust, and anti-immigrant attitudes. J. Soc. Issues 74, 774-789. doi: 10.1111/josi.12298

Mikolov, T., Sutskever, I., Chen, K., Corrado, G. S., and Dean, J. (2013). "Distributed representations of words and phrases and their compositionality," In Proceedings of the 26th International Conference on Neural Information Processing Systems, Vol.2 (Lake Tahoe, NV), 3111-3119.

Mohammad, S. M. (2018). "Obtaining reliable human ratings of valence, arousal, and dominance for 20,000 english words," in Proceedings of The Annual Conference of the Association for Computational Linguistics (ACL) (Melbourne, VIC). doi: 10.18653/v1/P18-1017

Monroe, B. L., Colaresi, M. P., and Quinn, K. M. (2008). Fightin'words: lexical feature selection and evaluation for identifying the content of political conflict. Polit. Anal. 16, 372-403. doi: 10.1093/pan/mpn018

Niculae, V., Suen, C., Zhang, J., Danescu-Niculescu-Mizil, C., and Leskovec, J. (2015). "Quotus: the structure of political media coverage as revealed by quoting patterns," in Proceedings of the 24th International Conference on World Wide Web (Florence: International World Wide Web Conferences Steering Committee), 798-808. doi: 10.1145/2736277.2741688

Nussbaum, M. C. (1999). Sex and Social Justice. Oxford: Oxford University Press. Opotow, S. (1990). Moral exclusion and injustice: an introduction. J. Soc. Issues 46, 1-20. doi: 10.1111/j.1540-4560.1990.tb00268.x

Osgood, C. E., Suci, G. J., and Tannenbaum, P. H. (1957). The Measurement of Meaning. Number 47. Chicago, IL: University of Illinois Press.

Ott, B. L., and Aoki, E. (2002). The politics of negotiating public tragedy: media framing of the matthew shepard murder. Rhetor. Public Affairs 5, 483-505. doi: 10.1353/rap.2002.0060

Palmer, A., Robinson, M., and Phillips, K. K. (2017). "Illegal is not a noun: linguistic form for detection of pejorative nominalizations," in Proceedings of the First Workshop on Abusive Language Online (Vancouver, CA), 91-100. doi: 10.18653/v1/W17-3014

Pennebaker, J. W., Francis, M. E., and Booth, R. J. (2001). Linguistic Inquiry and Word Count: Liwc 2001. Mahway, NJ: Lawrence Erlbaum Associates.

Peters, J. W. (2014). The Declineand Fall of the ' $h$ ' Word, New York: New York Times.

Peters, M. E., Neumann, M., Iyyer, M., Gardner, M., Clark, C., Lee, K., et al. (2018). "Deep contextualized word representations," in Proceedings of NAACL (New Orleans, LA). doi: 10.18653/v1/N18-1202

Pew Research Center (2017). Changing Attitudes on Gay Marriage. Available online at: http://www.pewforum.org/fact-sheet/changing-attitudes-on-gay-marriage/

Pryzant, R., Martinez, R. D., Dass, N., Kurohashi, S., Jurafsky, D., and Yang, D. (2019). Automatically neutralizing subjective bias in text. arXiv[Preprint].arXiv: 1911.09709. doi: 10.1609/aaai.v34i01.5385 
Rashkin, H., Singh, S., and Choi, Y. (2016). "Connotation frames: a data-driven investigation," in Proceedings of the 54th Annual Meeting of the Association for Computational Linguistics (Volume 1: Long Papers) (Berlin), 311-321. doi: $10.18653 / \mathrm{v} 1 / \mathrm{P} 16-1030$

Recasens, M., Danescu-Niculescu-Mizil, C., and Jurafsky, D. (2013). "Linguistic models for analyzing and detecting biased language," in Proceedings of the 51st Annual Meeting of the Association for Computational Linguistics (Volume 1: Long Papers) (Sofia), 1650-1659.

Rothbart, M., and Taylor, M. (1992). Category labels and social reality: Do we view social categories as natural kinds. Language, interaction and social cognition. London: Sage.

Rudinger, R., Naradowsky, J., Leonard, B., and Van Durme, B. (2018). “Gender bias in coreference resolution," in Proceedings of the 2018 Conference of the North American Chapter of the Association for Computational Linguistics: Human Language Technologies, Volume 2 (Short Papers) (New Orleans, LA), 8-14. doi: $10.18653 / \mathrm{v} 1 / \mathrm{N} 18-2002$

Russell, J. A. (1980). A circumplex model of affect. J. Pers. Soc. Psychol. 39:1161. doi: $10.1037 /$ h0077714

Sap, M., Gabriel, S., Qin, L., Jurafsky, D., Smith, N. A., and Choi, Y. (2019). Social bias frames: reasoning about social and power implications of language. arXiv[Preprint].arXiv: 1911.03891.

Sap, M., Prasettio, M. C., Holtzman, A., Rashkin, H., and Choi, Y. (2017). "Connotation frames of power and agency in modern films," in Proceedings of the 2017 Conference on Empirical Methods in Natural Language Processing (Copenhagen), 2329-2334. doi: 10.18653/v1/D17-1247

Schmidt, A., and Wiegand, M. (2017). "A survey on hate speech detection using natural language processing," in Proceedings of the Fifth International Workshop on Natural Language Processing for Social Media (Valencia), 1-10. doi: 10.18653/v1/W17-1101

Shah, N. (2019). "Asians are good at math" is not a compliment: stem success as a threat to personhood. Harvard Educ. Rev. 89, 661-686. doi: 10.17763/1943-5045-89.4.661

Sherman, G. D., and Haidt, J. (2011). Cuteness and disgust: the humanizing and dehumanizing effects of emotion. Emot. Rev. 3, 245-251. doi: $10.1177 / 1754073911402396$

Shuman, E. L. (1894). Steps Into Journalism: Helps and Hints for Young Writers. Evanston, IL: Correspondence School of Journalism.

Silva, L., Mondal, M., Correa, D., Benevenuto, F., and Weber, I. (2016). “Analyzing the targets of hate in online social media," in Tenth International AAAI Conference on Web and Social Media (Cologne: AAAI), 687-690.

Smith, B. A., Murib, Z., Motta, M., Callaghan, T. H., and Theys, M. (2017). "Gay" or "homosexual"? The implications of social category labels for the structure of mass attitudes. Am. Polit. Res. 46:1532673X17706560. doi: $10.1177 / 1532673 X 17706560$

Soller, K. (2018). Six Times Journalists on the Paper's History of Covering Aids and Gay Issues, New York: New York Times.

Steuter, E., and Wills, D. (2010). 'The vermin have struck again': dehumanizing the enemy in post 9/11 media representations. Media War Conflict 3, 152-167. doi: $10.1177 / 1750635210360082$

Sun, T., Gaut, A., Tang, S., Huang, Y., ElSherief, M., Zhao, J., et al. (2019). "Mitigating gender bias in natural language processing: literature review," in Proceedings of the 57th Annual Meeting of the Association for Computational Linguistics (Florence), 1630-1640. doi: 10.18653/v1/ P19-1159
Tipler, C., and Ruscher, J. B. (2014). Agency's role in dehumanization: nonhuman metaphors of out-groups. Soc. Pers. Psychol. Compass 8, 214-228. doi: $10.1111 / \mathrm{spc} 3.12100$

Tsur, O., Calacci, D., and Lazer, D. (2015). "A frame of mind: Using statistical models for detection of framing and agenda setting campaigns," in Proceedings of the 53rd Annual Meeting of the Association for Computational Linguistics and the 7th International Joint Conference on Natural Language Processing (Volume 1: Long Papers) (Beijing), 1629-1638. doi: 10.3115/v1/P15-1157

Tsvetkov, Y., Boytsov, L., Gershman, A., Nyberg, E., and Dyer, C. (2014). "Metaphor detection with cross-lingual model transfer," in Proceedings of the 52nd Annual Meeting of the Association for Computational Linguistics (Volume 1: Long Papers) (Baltimore, MD), 248-258. doi: 10.3115/v1/P14-1024

Van Bavel, J. J., Baicker, K., Boggio, P. S., Capraro, V., Cichocka, A., Cikara, M., et al. (2020). Using social and behavioural science to support covid-19 pandemic response. Nat. Hum. Behav. 4, 460-471. doi: $10.1038 /$ s41562-020-0884-Z

Vidgen, B., Botelho, A., Broniatowski, D., Guest, E., Hall, M., Margetts, H., et al. (2020). Detecting east asian prejudice on social media. arXiv[Preprint].arXiv: 2005.03909.

Voigt, R., Camp, N. P., Prabhakaran, V., Hamilton, W. L., Hetey, R. C., Griffiths, C. M., et al. (2017). Language from police body camera footage shows racial disparities in officer respect. Proc. Natl. Acad. Sci. U.S.A. 114, 6521-6526. doi: $10.1073 /$ pnas. 1702413114

Wang, Z., and Potts, C. (2019). "Talkdown: a corpus for condescension detection in context," in Proceedings of the 2019 Conference on Empirical Methods in Natural Language Processing and the 9th International Joint Conference on Natural Language Processing (EMNLP-IJCNLP) (Hong Kong), 3702-3710. doi: 10.18653/v1/D19-1385

Wiebe, J., Wilson, T., Bruce, R., Bell, M., and Martin, M. (2004). Learning subjective language. Comput. Linguist. 30, 277-308. doi: 10.1162/0891201041850885

Wierzbicka, A. (1986). What's in a noun? (or: how do nouns differ in meaning from adjectives?). Stud. Lang. 10, 353-389. doi: 10.1075/sl.10.2.05wie

$\mathrm{Xu}$, C., and Liu, M. Y. (2020). Social Cost With No Political Gain: The "Chinese Virus" Effect.

Zhao, J., Wang, T., Yatskar, M., Ordonez, V., and Chang, K.-W. (2018). "Gender bias in coreference resolution: evaluation and debiasing methods," in Proceedings of the 2018 Conference of the North American Chapter of the Association for Computational Linguistics: Human Language Technologies, Vol.2 (New Orleans, LA). doi: 10.18653/v1/N18-2003

Ziems, C., He, B., Soni, S., and Kumar, S. (2020). Racism is a virus: antiasian hate and counterhate in social media during the covid-19 crisis. arXiv[Preprint].arXiv: 2005.12423.

Conflict of Interest: The authors declare that the research was conducted in the absence of any commercial or financial relationships that could be construed as a potential conflict of interest.

Copyright (C) 2020 Mendelsohn, Tsvetkov and Jurafsky. This is an open-access article distributed under the terms of the Creative Commons Attribution License (CC BY). The use, distribution or reproduction in other forums is permitted, provided the original author(s) and the copyright owner(s) are credited and that the original publication in this journal is cited, in accordance with accepted academic practice. No use, distribution or reproduction is permitted which does not comply with these terms. 